DESY 07-212

December 2007

\title{
Theoretical Interest in $B$-Meson Physics at the B Factories, Tevatron and the $\mathrm{LHC}^{*}$
}

\author{
Ahmed Ali \\ Deutsches Elektronen-Synchrotron DESY, Hamburg, Germany \\ E-mail: ahmed.ali@desy.de
}

\begin{abstract}
We review the salient features of $B$-meson physics, with particular emphasis on the measurements carried out at the $B$-factories and Tevatron, theoretical progress in understanding these measurements in the context of the standard model, and anticipation at the LHC. Topics discussed specifically are the current status of the Cabibbo-Kobayashi-Maskawa matrix, the CP-violating phases, rare radiative and semileptonic decays, and some selected non-leptonic two-body decays of the $B$ mesons.
\end{abstract}

\section{Introduction}

The two $B$-meson factories operating at the KEK and SLAC $e^{+} e^{-}$storage rings have outperformed their projected luminosities and have produced a wealth of data on the process $e^{+} e^{-} \rightarrow \Upsilon(4 S) \rightarrow B \bar{B}$, with subsequent weak decays of the $B$ and $\bar{B}$ mesons. The exclusivity of the final state, the asymmetric beam energies and the large statistics collected at the $\Upsilon(4 S)$ $\left(O\left(10^{9}\right) B \bar{B}\right.$ events) have led to a number of impressive and quantitative results, which include, among other measurements, precise determination of the weak mixing (Cabibbo-Kobayashi-Maskawa CKM ${ }^{1,2}$ ) matrix elements $\left|V_{c b}\right|$ and $\left|V_{u b}\right|^{3}$, a large number of CP-violating asymmetries in exclusive decays of the $B$ mesons ${ }^{4}$, and rare radiative and semileptonic decays (implying flavor changing neutral current FCNC transitions) involving the $B^{0}$ and $B^{+}$mesons and their charge conjugates ${ }^{5}$. Recently, Belle collaboration have published a number of interesting results on the decays of

*Invited Talk, International Symposium on Contemporary Physics, National Centre for Physics, Islamabad, Pakistan (March 26-30, 2007); to be published in the symposium proceedings. 
the $B_{s}$-meson highlighted by the first measurement of the radiative penguin decay $B_{s} \rightarrow \phi \gamma{ }^{6}$. This is the $\mathrm{SU}(3)$-counterpart to the much celebrated decay $B \rightarrow K^{*} \gamma$, first measured by CLEO and further consolidated by the BABAR and BELLE collaborations ${ }^{7}$.

The two B-meson factories are not the only players currently active in the study of $B$-meson physics. In fact, $b$-physics is the main beneficiary of the upgrade program at the high energy proton-antiproton collider Tevatron at Fermilab. Since this upgrade, physics news from Fermilab are dominated by the achievements in the $b$-quark sector. We have seen very impressive and seminal results in this field by the two Fermilab experiments CDF and D0, including the landmark measurement of the $B_{s}^{0}-\overline{B_{s}^{0}}$-mixing induced mass difference $\Delta M_{B_{s}} 8,9$ and the first result on direct $\mathrm{CP}$ violation in the $B_{s}$-meson sector $\mathcal{A}_{\mathrm{CP}}\left(B_{s}^{0} \rightarrow K^{+} \pi^{-}\right)$10, 11. These experiments have established (if any proof was needed) that cutting edge flavor physics is done also at hadron machines. In all likelihood, this success story of the hadron machines will be set forth at the Large Hadron Collider (LHC), commissioned to operate in 2008 at CERN. In particular, the experiment $\mathrm{LHCb}^{12}$, dedicated to precision measurements of the physics of the entire family of $B$-mesons $\left(B^{0}, B^{+}, B_{s}^{0}, B_{c}^{+}\right.$, their charged conjugated states, and excited states) and $\Lambda_{b}$ baryons (including the entire baryon spectrum containing at least one $b$-quark), will greatly broaden our knowledge of both the spectroscopic and dynamical aspects of $b$-physics. The other two LHC experiments ATLAS and CMS will also contribute to $b$ physics.

In this talk, I will briefly review the highlights of the measurements already accomplished, making contacts with theoretical expectations in the standard model (SM). The topics discussed are: (i) an update of the CKM matrix, with emphasis on the matrix elements in the third row and the third column of $V_{\mathrm{CKM}}$ from direct decays (which determine $\left|V_{c b}\right|,\left|V_{u b}\right|$ and $\left|V_{t b}\right|$ ), and from induced transitions involving the mass differences $\Delta M_{B_{d}}$, $\Delta M_{B_{s}}$ and the electromagnetic penguin decays $b \rightarrow(s, d) \gamma$ (which determine the matrix elements $\left|V_{t d}\right|$ and $\left|V_{t s}\right|$ ) (ii) measurements of the CPviolating phases $\alpha, \beta$ and $\gamma$ from exclusive $B$-decays, and (iii) radiative, semileptonic and leptonic rare $B$-decays, a field which has received a lot of theoretical attention both in the context of the SM and in extensions of it. Much more detailed discussions of these topics can be found in the original literature cited below, in the reviews, such as the Review of Particle Properties by the Particle Data Group ${ }^{13}$, and in the proceedings of the topical workshops on flavor physics 14 . 


\section{Status of the CKM Matrix}

The CKM matrix is written below in the Wolfenstein parameterization 15 in terms of the four parameters $A, \lambda, \rho, \eta$ :

$$
V_{\mathrm{CKM}} \equiv\left(\begin{array}{ccc}
1-\frac{1}{2} \lambda^{2} & \lambda & A \lambda^{3}(\rho-i \eta) \\
-\lambda\left(1+i A^{2} \lambda^{4} \eta\right) & 1-\frac{1}{2} \lambda^{2} & A \lambda^{2} \\
A \lambda^{3}(1-\rho-i \eta) & -A \lambda^{2}\left(1+i \lambda^{2} \eta\right) & 1
\end{array}\right) .
$$

Anticipating precision data, a perturbatively improved version 16 of the Wolfenstein parameterization will be used below with $\bar{\rho}=\rho\left(1-\lambda^{2} / 2\right), \quad \bar{\eta}=$ $\eta\left(1-\lambda^{2} / 2\right)$.

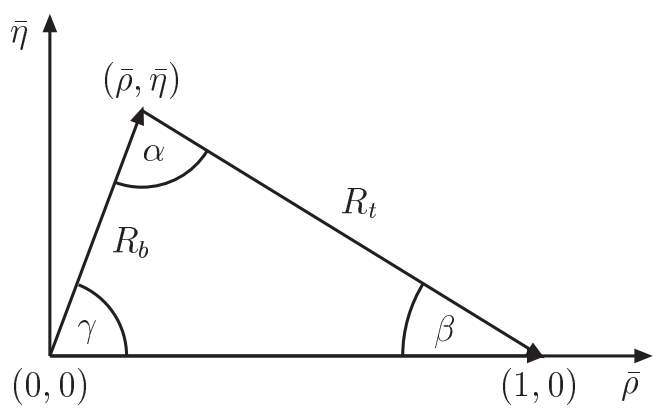

Fig. 1. The unitarity triangle with unit base in the $\bar{\rho}-\bar{\eta}$ plane.

Unitarity of the CKM matrix implies six relations, of which the one resulting from the equation $V_{u d} V_{u b}^{*}+V_{c d} V_{c b}^{*}+V_{t d} V_{t b}^{*}=0$ is the principal focus of the current experiments in $B$-decays. This is a triangle relation in the complex plane (i.e. $\bar{\rho}-\bar{\eta}$ space), and the three angles of this triangle are called $\alpha, \beta$ and $\gamma$, with the BELLE convention being $\phi_{1}=\beta, \phi_{2}=\alpha$ and $\phi_{3}=\gamma$ (see Fig.1). The unitarity relation in discussion can also be written as

$$
R_{b} \mathrm{e}^{i \gamma}+R_{t} \mathrm{e}^{-i \beta}=1,
$$

where $R_{b}=\left(1-\frac{\lambda^{2}}{2}\right) \frac{1}{\lambda}\left|\frac{V_{u b}}{V_{c b}}\right|=\sqrt{\bar{\rho}^{2}+\bar{\eta}^{2}}$ and $R_{t}=\frac{1}{\lambda}\left|\frac{V_{t d}}{V_{c b}}\right|=$ $\sqrt{(1-\bar{\rho})^{2}+\bar{\eta}^{2}}$. Thus, precise determination of $\left|V_{c b}\right|,\left|V_{u b}\right|$ and $\left|V_{t d}\right|$ and the three CP-violating phases $\alpha, \beta, \gamma$ is crucial in testing the CKM paradigm.

In this section, we mainly review the current status of the CKM matrix elements in the third row and the third column, namely $V_{u b}, V_{c b}, V_{t d}, V_{t s}$ 
and $V_{t b}$. The other four CKM matrix elements, $V_{u d}, V_{u s}, V_{c d}$ and $V_{c s}$ are discussed in 13,3 , from where we quote the current best measurements.

- $V_{u d}$ is obtained from the nuclear $\beta$-decay of the (super-allowed) $O^{+} \rightarrow O^{+}$transitions 17,18 , yielding: $V_{u d}=0.97377(27)$.

- $V_{u s}$ is obtained from the $K_{\ell 3}$ decays and chiral perturbation theory, yielding $V_{u s}=0.22535 \pm 0.00116$. In getting this, the LeutwylerRoos value for the form factor $f_{+}(0)=0.961 \pm 0.008{ }^{19}$ has been used. This is to be contrasted with the value, $V_{u s}=\lambda=0.2265(7)$, obtained from the unitarity condition on the first row of the CKM matrix and the input value for $V_{u d}$ given above. They are in good agreement with each other, providing a precision test of the unitarity of the first row of $V_{\mathrm{CKM}}$.

- $V_{c s}$ is obtained from the decay $D \rightarrow K e \nu_{e}$ and the Lattice-QCD result for the $D \rightarrow K$ form factor ${ }^{20}$, yielding $\left|V_{c s}\right|=0.996 \pm$ $0.008 \pm 0.015 \pm 0.104$, where the first two errors are experimental and the third (dominant) error is from theory.

- Despite impressive progress in the measurement of the Cabibbosuppressed $D$-meson decays and Lattice-QCD, the determination of $V_{c d}$ is still dominated by the neutrino-nucleon production of the charm quark, yielding: $\left|V_{c d}\right|=0.230 \pm 0.011^{13}$. Within errors, $\left|V_{c d}\right|=\left|V_{u s}\right|$, as anticipated from eq. (1).

\subsection{Current determinations of $\left|V_{c b}\right|$ and $\left|V_{u b}\right|$}

Determinations of $\left|V_{c b}\right|$ are based on the semileptonic decay $b \rightarrow c \ell \nu_{\ell}$. This transition can be measured either inclusively through the process $B \rightarrow X_{c} \ell \nu_{\ell}$, where $X_{c}$ is a hadronic state with a net $c$-quantum number, or exclusively, such as the decays $B \rightarrow\left(D, D^{*}\right) \ell \nu_{\ell}$. In either case, intimate knowledge of QCD is required to go from the partonic process to

the hadronic states. The fact that $m_{b} \gg \Lambda_{\mathrm{QCD}}$ has led to novel applications of QCD in which heavy quark expansion (HQE) plays a central role 21 and the underlying theory is termed as HQET.

\subsection{1. $\left|V_{c b}\right|$ from the decays $B \rightarrow X_{c} \ell \nu_{\ell}$}

Concentrating first on the inclusive decays, the semileptonic decay rate can be calculated as a power series

$$
\Gamma=\Gamma_{0}+\frac{1}{m_{b}} \Gamma_{1}+\frac{1}{m_{b}^{2}} \Gamma_{2}+\frac{1}{m_{b}^{3}} \Gamma_{3}+\ldots,
$$


where each $\Gamma_{i}$ is a perturbation series in $\alpha_{s}\left(m_{b}\right)$, the QCD coupling constant at the scale $m_{b}$. Here $\Gamma_{0}$ is the decay width of a free $b$-quark, which gives the parton-model result. The coefficient of the leading power correction $\Gamma_{1}$ is absent ${ }^{22}$, and the effect of the $1 / m_{b}^{2}$ correction is collected in $\Gamma_{2}$, which can be expressed in terms of two non-perturbative parameters called $\lambda_{1}$ kinetic energy of the $b$-quark - and $\lambda_{2}$ - its chromomagnetic moment. These quantities, also called $\mu_{\pi}^{2}$ and $\mu_{G}^{2}$, respectively, in the literature, are defined in terms of the following matrix elements $23,24,25,26$ :

$$
\begin{aligned}
& 2 M_{B} \lambda_{1} \equiv\left\langle B(v)\left|\bar{Q}_{v}(i D)^{2} Q_{v}\right| B(v)\right\rangle, \\
& 6 M_{B} \lambda_{2} \equiv\left\langle B(v)\left|\bar{Q}_{v} \sigma_{\mu \nu}\left[i D^{\mu}, i D^{\nu}\right] Q_{v}\right| B(v)\right\rangle,
\end{aligned}
$$

where $D_{\mu}$ is the covariant derivative and heavy quark fields are characterized by the 4 -velocity, $v$. At $\mathcal{O}\left(\Lambda_{\mathrm{QCD}}^{3} / m_{b}^{3}\right)$, six new matrix elements enter in $\Gamma_{3}$, usually denoted by $\rho_{1,2}$ and $\mathcal{T}_{1,2,3,4}$.

Data have been analyzed in the theoretical accuracy in which corrections up to $\mathcal{O}\left(\alpha_{s}^{2} \beta_{0}\right), \mathcal{O}\left(\alpha_{s} \Lambda_{\mathrm{QCD}} / m_{b}\right)$ and $\mathcal{O}\left(\Lambda_{\mathrm{QCD}}^{3} / m_{b}^{3}\right)$ are taken into account 27,28 , with $\beta_{0}$ being the lowest order coefficient in the expansion of the QCD $\beta$-function. In addition to the other parameters, a quark mass scheme has to be specified. Bauer et al. ${ }^{27}$ have carried out a comprehensive study of the scheme dependence using five quark mass schemes: $1 S$, $\mathrm{PS}, \overline{\mathrm{MS}}$, kinematic, and the pole mass.

To extract the value of $\left|V_{c b}\right|$ and other fit parameters, three different distributions, namely the charged lepton energy spectrum and the hadronic invariant mass spectrum in $B \rightarrow X_{c} \ell \bar{\nu}_{\ell}$, and the photon energy spectrum in $B \rightarrow X_{s} \gamma$ have been studied. Theoretical analyses are carried out in terms of the moments. Defining the integral $R_{n}\left(E_{\text {cut }}, \mu\right) \equiv$ $\int_{E_{\text {cut }}} d E_{\ell}\left(E_{\ell}-\mu\right)^{n} d \Gamma / d E_{\ell}$, where $E_{\text {cut }}$ is a lower cut on the charged lepton energy, moments of the lepton energy spectrum are given by $\left\langle E_{\ell}^{n}\right\rangle=$ $R_{n}\left(E_{\text {cut }}, 0\right) / R_{0}\left(E_{\text {cut }}, 0\right)$. For the $B \rightarrow X_{c} \ell \bar{\nu}_{\ell}$ hadronic invariant mass spectrum, the moments are defined likewise with the cutoff $E_{\text {cut }}$. Analyses of the data along these lines have been presented by a large number of experiments. For a summary and references to the original literature, see HFAG ${ }^{7}$.

The global fit of the world data in the so-called $1 S$-scheme for the $b$ quark mass undertaken by Bauer et al. ${ }^{27}$ leads to the following fit values for $\left|V_{c b}\right|$ and $m_{b}^{1 S}$ :

$$
\begin{aligned}
& \left|V_{c b}\right|=\left(41.78 \pm 0.30 \pm 0.08_{\tau_{B}}\right) \times 10^{-3}, \\
& m_{b}^{1 S}=(4.701 \pm 0.030) \mathrm{GeV} .
\end{aligned}
$$

The BABAR collaboration have studied the dependence of the lepton and 
hadron moments on the cutoff $E_{\text {cut }}$ and compared their measurements with the theoretical calculation by Gambino and Uraltsev 28 using the so-called kinematic scheme for the $b$-quark mass $m_{b}^{\text {kin }}(\mu)$, renormalized at the scale $\mu=1 \mathrm{GeV}$. Agreement between experiment and theory allows to determine the fit parameters in this scheme with the results 7 :

$$
\begin{aligned}
& \left|V_{c b}\right|=\left(41.91 \pm 0.19_{\exp } \pm 0.28_{H Q E} \pm 0.59_{\Gamma_{\mathrm{sl}}}\right) \times 10^{-3} \\
& m_{b}(1 \mathrm{GeV})=\left(4.613 \pm 0.022_{\exp } \pm 0.027_{H Q E}\right) \mathrm{GeV} \\
& m_{c}(1 \mathrm{GeV})=\left(1.187 \pm 0.033_{\text {exp }} \pm 0.040_{H Q E}\right) \mathrm{GeV}
\end{aligned}
$$

The two analyses (5) and (6) are in excellent agreement with each other. The achieved accuracy $\delta\left|V_{c b}\right| /\left|V_{c b}\right| \simeq 2 \%$ is impressive, and the precision on $m_{b}$ is also remarkable, $\delta m_{b} / m_{b}=O\left(10^{-3}\right)$, with a similar precision obtained on the mass difference $m_{b}-m_{c}$.

\section{2. $\left|V_{c b}\right|$ from $B \rightarrow\left(D, D^{*}\right) \ell \nu_{\ell}$ decays}

The classic application of HQET in heavy $\rightarrow$ heavy decays is in the decay $B \rightarrow D^{*} \ell \nu_{\ell}$. The differential distribution in the variable $\omega\left(=v_{B} \cdot v_{D^{*}}\right)$, where $v_{B}\left(v_{D^{*}}\right)$ is the four-velocity of the $B\left(D^{*}\right)$-meson, is given by

$$
\frac{d \Gamma}{d \omega}=\frac{G_{F}^{2}}{4 \pi^{3}}\left|V_{c b}\right|^{2} m_{D^{*}}^{3}\left(m_{B}-m_{D^{*}}\right)^{2}\left(\omega^{2}-1\right)^{1 / 2} \mathcal{G}(\omega)|\mathcal{F}(\omega)|^{2},
$$

where $\mathcal{G}(\omega)$ is a phase space factor with $\mathcal{G}(1)=1$, and $\mathcal{F}(\omega)$ is the IsgurWise (IW) function 29 with the normalization at the symmetry point $\mathcal{F}(1)=1$. Leading $\Lambda_{\mathrm{QCD}} / m_{b}$ corrections in $\mathcal{F}(1)$ are absent due to Luke's theorem 30 . Theoretical issues are the precise determination of the second order power correction to $\mathcal{F}(\omega=1)$, the slope $\rho^{2}$ and the curvature $c$ of the IW-function:

$$
\mathcal{F}(\omega)=\mathcal{F}(1)\left[1-\rho^{2}(\omega-1)+c(\omega-1)^{2}+\ldots\right] .
$$

Bounds on $\rho^{2}$ have been obtained by Bjorken 31 and Uraltsev ${ }^{32}$, which can be combined to yield $\rho^{2}>3 / 4$. Likewise, bounds on the second (and higher) derivatives of the IW-function have been worked out by the Orsay group 33 , yielding $c>15 / 32$. Apparently, the data sets used in this analysis differ significantly from experiment to experiment, resulting in considerable dispersion in the values of $\mathcal{F}(1)\left|V_{c b}\right|$ and $\rho^{2}$ and hence in a large $\chi^{2}$ of the combined fit, summarized by $\mathrm{HFAG}^{7}$ :

$$
\begin{aligned}
\mathcal{F}(1)\left|V_{c b}\right| & =(35.89 \pm 0.56) \times 10^{-3}, \\
\rho^{2} & =1.23 \pm 0.05 \quad\left(\chi^{2}=37.8 / 17 ; \mathrm{CL}=0.026\right) .
\end{aligned}
$$


To convert this into a value of $\left|V_{c b}\right|$, we need to know $\mathcal{F}(1)$. In terms of the perturbative (QED and QCD) and non-perturbative (leading $\delta_{1 / m^{2}}$ and sub-leading $\left.\delta_{1 / m^{3}}\right)$ corrections, $\mathcal{F}(1)$ can be expressed as follows:

$$
\mathcal{F}(1)=\eta_{A}\left[1+\delta_{1 / m^{2}}+\delta_{1 / m^{3}}\right]
$$

where $\eta_{A}$ is the perturbative renormalization of the IW-function, known in the meanwhile to three loops 34 . One- and two-loop corrections yield $\eta_{A} \simeq 0.933$ and the $O\left(\alpha_{s}^{3}\right)$ contribution amounts to $\eta_{A}^{(3)}=-0.005$. A Lattice-QCD calculation in the quenched approximation yields $35 \mathcal{F}(1)=$ $0.919_{-0.035}^{+0.030}$, which is now being reevaluated with dynamical quarks. Taking into account this theoretical input, the value quoted at the Lepton-Photon2007 Symposium is ${ }^{3}$ :

$$
\left|V_{c b}\right|_{B \rightarrow D^{*} \ell \nu_{\ell}}=\left(39.1 \pm 0.65_{\exp } \pm 1.4_{\text {theo }}\right) \times 10^{-3} .
$$

The resulting value of $\left|V_{c b}\right|$ is in excellent agreement with the ones given in (5) and (6) obtained from the inclusive decays.

\section{3. $\left|V_{u b}\right|$ from the decays $B \rightarrow X_{u} \ell \nu_{\ell}$}

HQET techniques allow to calculate the inclusive decay rate $B \rightarrow X_{u} \ell \nu_{\ell}$ rather accurately. However, the experimental problem in measuring this transition lies in the huge background from the dominant decays $B \rightarrow$ $X_{c} \ell \nu_{\ell}$ which can be brought under control only through severe cuts on the kinematics. For example, these cuts are imposed on the lepton energy, demanding $E_{\ell}>\left(m_{B}^{2}-m_{D}^{2}\right) / 2 m_{B}$, and/or the momentum transfer to the lepton pair $q^{2}$ restricting it below a threshold value $q^{2}<q_{\max }^{2}$, and/or the hadron mass recoiling against the leptons, which is required to satisfy $m_{X}<m_{D}$. With these cuts, the phase space of the decay $B \rightarrow X_{u} \ell \nu_{\ell}$ is greatly reduced. A bigger problem is encountered in the end-point region (also called the shape function region), where the leading power correction is no longer $1 / m_{b}^{2}$ but rather $1 / m_{b} \Lambda_{\mathrm{QCD}}$, slowing the convergence of the expansion. Moreover, in the region of energetic leptons with low invariant mass hadronic states, the differential rate is sensitive to the details of the shape function $f\left(k_{+}\right)^{36}$, where $k_{+}=k_{0}+k_{3}$ with $k^{\mu} \sim O\left(\Lambda_{\mathrm{QCD}}\right)$.

The need to know $f\left(k_{+}\right)$can be circumvented to a large extent by doing a combined analysis of the data on $B \rightarrow X_{u} \ell \nu_{\ell}$ and $B \rightarrow X_{s} \gamma$. Using the operator product expansion (OPE) to calculate the photon energy spectrum in the inclusive decay $B \rightarrow X_{s} \gamma$, the leading terms in the spectrum (neglecting the bremsstrahlung corrections) can be re-summed into a shape 
function 37 :

$$
\frac{d \Gamma_{s}}{d x}=\frac{G_{F}^{2} \alpha m_{b}^{5}}{32 \pi^{4}}\left|V_{t s} V_{t b}^{*}\right|^{2}\left|C_{7}^{\mathrm{eff}}\right|^{2} f(1-x),
$$

where $x=\frac{2 E_{\gamma}}{m_{b}}$, and $C_{7}^{\text {eff }}$ is an effective Wilson coefficient, characterizing the strength of the electromagnetic dipole operator. In the leading order, $E_{\ell^{-}}$ and $M_{X_{u}}$-spectra in $B \rightarrow X_{u} \ell \nu_{\ell}$ are also governed by $f(x)$. Thus, $f(x)$ can be measured in $B \rightarrow X_{s} \gamma$ and used in the analysis of data in $B \rightarrow X_{u} \ell \nu_{\ell}$.

Following this argument, a useful relation emerges $38,39,40$

$$
\left|\frac{V_{u b}}{V_{t b} V_{t s}^{*}}\right|=\left(\frac{3 \alpha}{\pi}\left|C_{7}^{\mathrm{eff}}\right|^{2} \frac{\Gamma_{u}\left(E_{c}\right)}{\Gamma_{s}\left(E_{c}\right)}\right)^{\frac{1}{2}}\left(1+\delta\left(E_{c}\right)\right),
$$

where

$$
\begin{aligned}
& \Gamma_{u}\left(E_{c}\right) \equiv \int_{E_{c}}^{m_{B} / 2} d E_{\ell} \frac{d \Gamma_{u}}{d E_{\ell}}, \\
& \Gamma_{s}\left(E_{c}\right) \equiv \frac{2}{m_{b}} \int_{E_{c}}^{m_{B} / 2} d E_{\gamma}\left(E_{\gamma}-E_{c}\right) \frac{d \Gamma_{s}}{d E_{\gamma}},
\end{aligned}
$$

and $\delta\left(E_{c}\right)$ incorporates the sub-leading terms in $\mathcal{O}\left(\Lambda_{\mathrm{QCD}} / m_{b}\right)$, which can only be modeled at present. In addition, there are perturbative corrections to the spectra and in the relation (11) $37,41,38$.

The other strategy is to extend the measurements of the inclusive decay $B \rightarrow X_{u} \ell^{+} \nu_{\ell}$ to the kinematic regions which are reachable by the decay $B \rightarrow X_{c} \ell^{+} \nu_{\ell}$, thus enlarging the region where the light-cone momentum component satisfies $P_{+} \equiv E_{X}-\left|\vec{P}_{X}\right| \gg \Lambda_{\mathrm{QCD}}$, obviating the need to know the shape function. Both these methods have been used in the determination of $\left|V_{u b}\right|$, summarized below.

- Determination of $\left|V_{u b}\right|$ using the combined cuts on the variables $m_{X}$ and $q^{2}$ following the suggestion by Bauer, Ligeti and Luke (BLL) ${ }^{42}$. Taking the HQE parameter input from the analysis of the decay $B \rightarrow X_{c} \ell \nu_{\ell}$ and $B \rightarrow X_{s} \gamma$, HFAG $^{7}$ quotes an average value $\left|V_{u b}\right|(\mathrm{BLL})=(4.83 \pm 0.24 \pm$ $0.37) \times 10^{-3}$, using the b-quark mass $m_{b}(1 \mathrm{~S})=(4.70 \pm 0.03) \mathrm{GeV}$ in the so-called $1 S$-scheme.

- Determination of $\left|V_{u b}\right|$ from a fully differential decay rate for $B \rightarrow X_{u} \ell \nu_{\ell}$ based on the soft collinear effective theory (SCET) techniques 43, 44, 45, 46, hereinafter called the Bosch-Lange-Neubert-Paz (BLNP) approach 47 . The three independent kinematic variables are chosen to be: $P_{\ell}=M_{B}-2 E_{\ell}, P_{-}=E_{X}+\left|\vec{P}_{X}\right|$, and $P_{+}=E_{X}-\left|\vec{P}_{X}\right|$, where $P_{ \pm}$ are the light-cone components of the hadronic final-state momentum along the jet direction, $E_{X}$ is the jet energy, $\vec{P}_{X}$ is the jet momentum, and $E_{\ell}$ is 
the charged-lepton energy. In terms of these variables, the triple differential distribution is:

$$
\begin{aligned}
\frac{d^{3} \Gamma}{d P_{+} d P_{-} d P_{\ell}}= & \frac{G_{F}^{2}\left|V_{u b}\right|^{2}}{16 \pi^{2}}\left(M_{B}-P_{+}\right)\left\{\left(P_{-}+P_{+}\right)\left(M_{B}-P_{-}+P_{\ell}-P_{+}\right) F_{1}\right. \\
& \left.+\left(M_{B}-P_{-}\right)\left(P_{-}-P_{+}\right) F_{2}+\left(P_{-}-P_{\ell}\right)\left(P_{\ell}-P_{+}\right) F_{3}\right\} \cdot(13)
\end{aligned}
$$

The "structure functions" $F_{i}$ can be expressed as product of the hard (perturbatively calculable) coefficient and a jet function, which are convoluted with the soft light-cone distribution functions, the shape functions of the $B$ meson. SCET allows to separate the two scales here, namely $\mu_{h} \sim m_{b}$ and $\mu_{i} \sim \sqrt{m_{b} \Lambda_{\mathrm{QCD}}}$ and enables to sum large logarithms involving the two scales $\mu_{h}$ and $\mu_{i}$. The dependence on the subleading shape functions is studied by taking several models. Fixing the HQE parameters (in the socalled shape function scheme) to the values $m_{b}(\mathrm{SF})=(4.63 \pm 0.06) \mathrm{GeV}$, $\mu_{\pi}^{2}(\mathrm{SF})=(0.18 \pm 0.06) \mathrm{GeV}^{2}$, and the exponential form for the shape function, HFAG ${ }^{7}$ quotes $\left|V_{u b}\right|=(4.31 \pm 0.17 \pm 0.35) \times 10^{-3}$ in the BLNP approach.

- Determination of $\left|V_{u b}\right|$ using the so-called Dressed Gluon Exponentiation (DGE) advocated by Andersen and Gardi ${ }^{48}$. The basic assumption of this approach is that properly defined quark distribution in an onshell heavy quark provides a good approximation to the distribution in the meson. The problematic small hadronic mass $M_{X_{u}}$ region in the decay $B \rightarrow X_{u} \ell^{+} \nu_{\ell}$ is characterized by a large hierarchy in the ratio $p_{j}^{+} / p_{j}^{-} \ll 1$ involving the partonic light-cone coordinates $p_{j}^{+} \leq p_{j}^{-} \leq m_{b}$. Defining the moments $n$ with respect to the powers of $1-p_{j}^{+} / p_{j}^{-}$, the region of small $p_{j}^{+}$ is probed by high moments $n \rightarrow \infty$, giving rise to the Sudakov logarithms $\ln n$. Infrared sensitivity appears in the moment-space Sudakov exponents through infrared renormalons, leading to the divergence of the (higher order perturbative) series in the Sudakov exponent. However, the leading infrared renormalon ambiguity cancels exactly against the pole-mass renormalon ambiguity, which enters as a kinematic factor in the differential decay width, making the on-shell perturbative calculation directly applicable for the phenomenology. This approach, applied to the data yields 7 $\left|V_{u b}\right|=(4.34 \pm 0.16 \pm 0.25) \times 10^{-3}$ for the input value $\overline{m_{b}}\left(\bar{m}_{b}\right)=(4.20 \pm 0.07)$ $\mathrm{GeV}$.

Thus, the three theoretical approaches used in the determination of $\left|V_{u b}\right|$ from the inclusive decays $B \rightarrow X_{u} \ell \nu_{\ell}$ give very consistent values. 


\section{4. $\left|V_{u b}\right|$ from exclusive decays}

$\left|V_{u b}\right|$ has also been determined from the exclusive decays $B \rightarrow \pi \ell \nu_{\ell}$. Theoretical accuracy is limited by the imprecise knowledge of the form factors. A number of theoretical techniques has been used to determine them. These include, among others, Light-cone QCD sum rules ${ }^{49}$, Quenched- and Unquenched-Lattice QCD simulations $50,51,52$. HFAG quotes the following extracted values of $\left|V_{u b}\right|$ using the full $q^{2}$-range for the form factors (in units of $\left.10^{-3}\right)^{7}$ :

$\left|V_{u b}\right|=3.43 \pm 0.10_{-0.42}^{+0.67}$ (LCSR: Ball-Zwicky ${ }^{49}$ );

$\left|V_{u b}\right|=3.17 \pm 0.10_{-0.48}^{+0.77}$ (Lattice: HPQCD ${ }^{50}$ );

$\left|V_{u b}\right|=3.82 \pm 0.12_{-0.52}^{+0.88}\left(\right.$ Lattice: FNAL $\left.{ }^{51}\right)$;

$\left|V_{u b}\right|=3.61 \pm 0.11_{-0.57}^{+1.11}$ (Lattice: $\mathrm{APE}^{52}$ ).

These values of $\left|V_{u b}\right|$ from the exclusive decays (typically $3.5 \times 10^{-3}$ ) are smaller than the ones extracted from the inclusive measurements (typically $\left.4.3 \times 10^{-3}\right)$. This mismatch is a mild irritant, waiting for its resolution through progress in Lattice-QCD and more $B$-factory data.

To reduce the form-factor related uncertainties in extracting $\left|V_{u b}\right|$ from exclusive decays $B \rightarrow(\pi, \rho) \ell \nu_{\ell}$, input from the rare $B$-decays $B \rightarrow$ $\left(K, K^{*}\right) \ell^{+} \ell^{-}$and HQET may be helpful. A proposal along these lines is the so-called Grinstein's double ratio which would determine $\left|V_{u b}\right| /\left|V_{t b} V_{t s}^{*}\right|$ from the end-point region of exclusive rare $B$-meson decays ${ }^{53}$. To carry out this program one has to measure four distributions in the decays $B \rightarrow \rho \ell \nu_{\ell}$, $B \rightarrow K^{*} \ell^{+} \ell^{-}$, and $D \rightarrow\left(\rho, K^{*}\right) \ell_{\ell}$. With the help of this data and HQET, the ratio of the CKM factors $\left|V_{u b}\right| /\left|V_{t b} V_{t s}^{*}\right|$ can be determined through the double ratio

$$
\frac{\Gamma(\bar{B} \rightarrow \rho \ell \nu)}{\Gamma\left(\bar{B} \rightarrow K^{*} \ell^{+} \ell^{-}\right)} \frac{\Gamma\left(D \rightarrow K^{*} \ell \nu\right)}{\Gamma(D \rightarrow \rho \ell \nu)} .
$$

At the $B$ factories, one expects enough data on these decays to allow a $10 \%$ determination of $\left|V_{u b}\right|$ from exclusive decays.

\section{Status of the Third Row of $V_{\mathrm{CKM}}$}

FCNC transitions $b \rightarrow s$ and $b \rightarrow d$ (as well as $s \rightarrow d$ ) give information on the third row of the CKM matrix $V_{\mathrm{CKM}}$ and allow to search for physics beyond the SM. In the SM these transitions are generally dominated by the (virtual) top quark contributions giving rise to the dependence on the matrix elements $\left|V_{t b}^{*} V_{t s}\right|$ (for $b \rightarrow s$ transitions) and $\left|V_{t b}^{*} V_{t d}\right|$ (for $b \rightarrow d$ transitions). Of these, only the matrix element $\left|V_{t b}\right|$ has been measured by a tree process $t \rightarrow W b$ at the Tevatron through the ratio 


$$
R_{t b} \equiv \frac{\mathcal{B}(t \rightarrow W b)}{\mathcal{B}(t \rightarrow W q)}=\frac{\left|V_{t b}\right|^{2}}{\left|V_{t d}\right|^{2}+\left|V_{t s}\right|^{2}+\left|V_{t b}\right|^{2}}
$$

The current measurements yield ${ }^{54}: R_{t b}=1.12_{-0.19}^{+0.21}$ (stat) $)_{-0.13}^{+0.17}$ (syst), yielding $R_{t b}>0.61$ (at $95 \%$ C.L.), which in turn gives $\left|V_{t b}\right|>0.78$ at $95 \%$ C.L..

Precision on the direct measurement of $\left|V_{t b}\right|$ is rather modest (unitarity gives $\left|V_{t b}\right| \simeq 0.9992$.), which will be greatly improved, in particular, at a Linear Collider 55 , but also at the LHC. The corresponding measurements of $\left|V_{t s}\right|$ and $\left|V_{t d}\right|$ from the tree processes are not on the cards. These matrix elements are determined by (loop) induced processes discussed below.

\subsection{Status of $\left|V_{t d}\right|$}

The current best measurement of $\left|V_{t d}\right|$ comes from $\Delta M_{B_{d}}$, the mass difference between the two mass eigenstates of the $B_{d}^{0}-\overline{B_{d}^{0}}$ complex. This has been measured in a number of experiments and is known to an accuracy of $\sim 1 \%$; the current world average is ${ }^{7} \Delta M_{B_{d}}=0.507 \pm 0.005(\mathrm{ps})^{-1}$.

In the SM, $\Delta M_{B_{d}}$ and its counterpart $\Delta M_{B_{s}}$, the mass difference in the $B_{s}^{0}-\overline{B_{s}^{0}}$ system, are calculated by box diagrams, dominated by the $W t$ loop. Since $\left(M_{W}, m_{t}\right) \gg m_{b}, \Delta M_{B_{d}}$ is governed by the short-distance physics. The expression for $\Delta M_{B_{d}}$ taking into account the perturbativeQCD corrections reads as follows 56

$$
\Delta M_{B_{d}}=\frac{G_{F}^{2}}{6 \pi^{2}} \hat{\eta}_{B}\left|V_{t d} V_{t b}^{*}\right|^{2} M_{B_{d}}\left(f_{B_{d}}^{2} \hat{B}_{B_{d}}\right) M_{W}^{2} S_{0}\left(x_{t}\right) .
$$

The quantity $\hat{\eta}_{B}$ is the next-to-leading log (NLL) perturbative QCD renormalization of the matrix element of the $(|\Delta B|=2, \Delta Q=0)$ four-quark operator, whose value is $\hat{\eta}_{B}=0.55 \pm 0.01 \quad 57 ; x_{t}=m_{t}^{2} / M_{W}^{2}$ and $S_{0}\left(x_{t}\right)=x_{t} f_{2}\left(x_{t}\right)$ is an Inami-Lim function ${ }^{58}$, with

$$
f_{2}(x)=\frac{1}{4}+\frac{9}{4} \frac{1}{(1-x)}-\frac{3}{2} \frac{1}{(1-x)^{2}}-\frac{3}{2} \frac{x^{2} \ln x}{(1-x)^{3}} .
$$

The quantity $f_{B_{d}}^{2} \hat{B}_{B_{d}}$ enters through the hadronic matrix element of the four-quark box operator, defined as:

$$
\left\langle\bar{B}_{q}^{0}\left|\left(\bar{b} \gamma_{\mu}\left(1-\gamma_{5}\right) q\right)^{2}\right| B_{q}^{0}\right\rangle \equiv \frac{8}{3} f_{B_{q}}^{2} B_{B_{q}} M_{B_{q}}^{2},
$$

with $B_{q}=B_{d}$ or $B_{s}$. With $\Delta M_{B_{d}}$ and $\hat{\eta}_{B}$ known to a high accuracy, and the current value of the top quark mass, defined in the $\overline{\mathrm{MS}}$ scheme, $\bar{m}_{t}\left(m_{t}\right)=$ $(162.3 \pm 2.2) \mathrm{GeV}$, leading to $S_{0}\left(x_{t}\right)=2.29(5)$, i.e., $\delta S_{0}\left(x_{t}\right) / S_{0}\left(x_{t}\right) \simeq 2.5 \%$, 
the combined error from these sources is a few percent. This is completely negligible in comparison with the current theoretical uncertainty on the matrix element $f_{B_{d}} \sqrt{\hat{B}_{B_{d}}}$. For example, $O\left(\alpha_{s}\right)$-improved calculations in the QCD sum rule approach yield $f_{B_{d}}=(210 \pm 19) \mathrm{MeV}^{59}$ and $f_{B_{d}}=(206 \pm 20)$ $\mathrm{MeV}^{60}$, whereas $\bar{B}_{B_{d}}$ in the $\overline{\mathrm{MS}}$ scheme in this approach is estimated as 61 $\bar{B}_{B_{d}}=1$ to within $10 \%$, yielding for the renormalization group invariant quantity $\hat{B}_{B_{d}} \simeq 1.46$, and an accuracy of about $\pm 15 \%$ on $f_{B_{d}} \sqrt{\hat{B}_{B_{d}}}$. Lattice calculations for $f_{B_{d}} \sqrt{\hat{B}_{B_{d}}}$ are uncertain due to the chiral extrapolation. Taking this into account, the current unquenched lattice QCD calculations from the JLQCD and HPQCD Collaborations yield 62,63

$$
f_{B_{d}} \sqrt{\hat{B}_{B_{d}}}=(244 \pm 11 \pm 24) \mathrm{MeV},
$$

where the first error is statistical, and the second combines the uncertainty from chiral extrapolation and other systematic errors. Using $\left|V_{t b}\right|=1$, this yields 64

$$
\left|V_{t d}\right|=(7.4 \pm 0.8) \times 10^{-3}
$$

\subsection{Present status of $\left|V_{t s}\right|$}

The quantity which currently provides the best determination of $\left|V_{t s}\right|$ is $\Delta M_{B_{s}}$, measured by D0 and CDF, with the CDF measurement being more precise $\Delta M_{B_{s}}=(17.77 \pm 0.10 \pm 0.07)(\mathrm{ps})^{-1} 11$. The expression for $\Delta M_{B_{s}}$ in the SM can be obtained from the one for $\Delta M_{B_{d}}$ (16) by the replacements: $M_{B_{d}} \rightarrow M_{B_{s}},\left(f_{B_{d}}^{2} \hat{B}_{B_{d}}\right) \rightarrow\left(f_{B_{s}}^{2} \hat{B}_{B_{s}}\right)$, and, most importantly, $V_{t d} \rightarrow V_{t s}$. Using the recent Lattice-QCD result $f_{B_{s}} \sqrt{\hat{B}_{B_{s}}}=(281 \pm 21) \mathrm{MeV}$ from the HPQCD collaboration 65 allows to determine $\left|V_{t s}\right|$ from $\Delta M_{B_{s}}$ with a precision of about $10 \%$, comparable to the precision on $\left|V_{t d}\right|$ in eq. (20). This is not competitive with the indirect estimate of this matrix element from the CKM unitarity, which yields $V_{t s} \simeq-V_{c b}=-4.1(1) \times 10^{-2}$.

The compatibility of the SM with the measured value of $\Delta M_{B_{s}}$ is usually tested by taking the value of $V_{t s}$ from the unitarity fits, the measured value of $m_{t}$, and the Lattice-QCD value for $f_{B_{s}} \sqrt{\hat{B}_{B_{s}}}$. Following this reasoning, the Lattice-HPQCD collaboration estimates $\Delta M_{B_{s}}(\mathrm{SM})=(20.3 \pm 3.0 \pm 0.8)$ $(\mathrm{ps})^{-1} 65$. The corresponding estimate from the UTfit is $\Delta M_{B_{s}}(\mathrm{SM})=$ $(20.9 \pm 2.6)(\mathrm{ps})^{-1} 66$ and the CKMfitter yields $\Delta M_{B_{s}}(\mathrm{SM})=\left(21.7_{-4.2}^{+5.9}\right)$ $(\mathrm{ps})^{-1} 67$. Thus, the experimental measurement of $\Delta M_{B_{s}}$ is typically about 
$1 \sigma$ below the SM estimates, with $\Delta M_{s}(\operatorname{expt}) / \Delta M_{s}(\mathrm{SM})=0.88 \pm 0.1365$, $0.85 \pm 0.10^{66}$ and $0.88 \pm 0.2067$.

Possible implications of the CDF measurement of $\Delta M_{B_{s}}$ have been studied in several papers $68,69,70,71,66$. Perhaps, it is to the point to mention here that a value of $\Delta M_{B_{s}} \simeq 18(\mathrm{ps})^{-1}$ was also hinted by the LEP data with the central value being $17.7(\mathrm{ps})^{-1}$. Anticipating this, the consequences of an eventual measurement of $\Delta M_{B_{s}}$ around this value were worked out some eight years ago ${ }^{72}$ for the parameters of the SM and the minimal flavor-violating supersymmetry ${ }^{73}$. It was emphasized that a measurement of $\Delta M_{B_{s}}$ around this value would confirm the SM. The CDF measurement of $\Delta M_{B_{s}}$ is very precise, and our knowledge of the CKM parameters and the non-perturbative quantities has in the meanwhile also improved, as discussed in this report. However, the bottom line remains the same, namely that the SM has passed this crucial test comfortably.

The ratio of the mass differences $\Delta M_{B_{d}} / \Delta M_{B_{s}}$, now measured very precisely, can be used to constrain the CKM ratio $\left|V_{t d}\right| /\left|V_{t s}\right|$ using the SM relation 74 :

$$
\frac{\Delta M_{B_{s}}}{\Delta M_{B_{d}}}=\xi \frac{M_{B_{s}}}{M_{B_{d}}} \frac{\left|V_{t b}^{*} V_{t s}\right|^{2}}{\left|V_{t b}^{*} V_{t d}\right|^{2}},
$$

where $\xi \equiv f_{B_{s}} \sqrt{\hat{B}_{B_{s}}} / f_{B_{d}} \sqrt{\hat{B}_{B_{d}}}$. Theoretical uncertainty in $\xi$ in the Lattice QCD approach is arguably smaller compared to the one in $f_{B_{s}} \sqrt{\hat{B}_{B_{s}}}$, as in the $\mathrm{SU}(3)$ limit $\xi=1$, and the uncertainty is actually in the $\mathrm{SU}(3)$-breaking corrections. Current estimate in the unquenched lattice calculations of $\xi$ is $64 \xi=1.21_{-0.035}^{+0.047}$, which yields 11

$$
\left|V_{t d} / V_{t s}\right|=0.2060 \pm 0.0007(\exp )_{-0.006}^{+0.008}(\mathrm{th}) .
$$

This is by far the best measurement of this CKM ratio, and it provides a non-trivial constraint on the allowed profile of the unitarity triangle. Combining eqs. (22) and (20) yields $\left|V_{t s}\right|=(36 \pm 4) \times 10^{-3}$ with the error dominated by theory. This completes our review of the CKM matrix elements $V_{i j}$.

\section{Radiative and Semileptonic Rare $B$ Decays}

Two inclusive rare $B$-decays of current experimental interest are $B \rightarrow X_{s} \gamma$ and $B \rightarrow X_{s} l^{+} l^{-}$, where $X_{s}$ is any charmless hadronic state with the strangeness quantum number $s=1$. They probe the SM in the electroweak $b \rightarrow s$ penguin sector. The CKM-suppressed decays $B \rightarrow X_{d} \gamma$ 
and $B \rightarrow X_{d} l^{+} l^{-}$are difficult to measure due to low rates and formidable backgrounds. Instead, the search for $B \rightarrow X_{d} \gamma$ decay has been carried out in the exclusive decay modes $B \rightarrow(\rho, \omega) \gamma$. Combined with the decay $B \rightarrow K^{*} \gamma$, these decays provide constraints on the CKM parameters. The CKM-suppressed decays $B \rightarrow(\pi, \rho, \omega) \ell^{+} \ell^{-}$have not yet been measured. We review some of these rare $B$-decays in the context of the SM.

\section{1. $B \rightarrow X_{s} \gamma: S M$ vs. Experiments}

The effective Lagrangian for the decays $B \rightarrow X_{s} \gamma$ obtained by integrating out the top quark and the heavy electroweak bosons reads as follows in the SM:

$$
\mathcal{L}_{\text {eff }}=\frac{4 G_{F}}{\sqrt{2}} V_{t s}^{*} V_{t b} \sum_{i=1}^{8} C_{i}(\mu) Q_{i}
$$

In writing this, unitarity of the CKM matrix has been used and the term proportional to the small matrix elements $V_{u s}^{*} V_{u b}$ has been ignored. The complete list of operators and their Wilson coefficients in the NNLO approximation evaluated at the scale $\mu=m_{b}$ can be seen elsewhere ${ }^{75}$.

The dominant four-quark operators $Q_{1}$ and $Q_{2}$ are

$$
Q_{1}=(\bar{s} c)_{V-A}(\bar{c} b)_{V-A}, \quad Q_{2}=\left(\bar{s}_{i} c_{j}\right)_{V-A}\left(\bar{c}_{j} b_{i}\right)_{V-A},
$$

and the electromagnetic and chromomagnetic penguin operators $Q_{7}$ and $Q_{8}$ are

$Q_{7}=-\frac{e \bar{m}_{b}(\mu)}{8 \pi^{2}}\left(\bar{s} \sigma^{\mu \nu}\left[1+\gamma_{5}\right] b\right) F_{\mu \nu}, \quad Q_{8}=-\frac{g \bar{m}_{b}(\mu)}{8 \pi^{2}}\left(\bar{s} \sigma^{\mu \nu}\left[1+\gamma_{5}\right] T^{a} b\right) G_{\mu \nu}^{a}$.

The factor $\overline{m_{b}}(\mu)$ is the $\overline{\mathrm{MS}}$ mass of the $b$ quark. QCD-improved calculations in the effective theory require three steps (for a review see ${ }^{76}$ ):

(i) Matching $C_{i}\left(\mu_{0}\right)\left(\mu_{0} \sim M_{W}, m_{t}\right)$ : They have been calculated up to three loops 77,78 . The three-loop matching is found to have less than $2 \%$ effect on $\mathcal{B}\left(B \rightarrow X_{s} \gamma\right){ }^{78}$.

(ii) Operator mixing: This involves calculation of the anomalous dimension matrix, which is expanded in $\alpha_{s}(\mu)$. The anomalous dimensions up to $\alpha_{s}^{2}(\mu)$ are known 79 since a decade, and the $\alpha_{s}^{3}(\mu)$ calculations have been completed recently in a series of papers $80,81,82$.

(iii) Matrix elements $\left\langle O_{i}\right\rangle\left(\mu_{b}\right)\left(\mu_{b} \sim m_{b}\right)$ : The first two terms in the expansion in $\alpha_{s}\left(\mu_{b}\right)$ are known since long 83 . Exact results to $\mathcal{O}\left(\alpha_{s}^{2}\right)$ were obtained for $Q_{7}$ in 84,85 and for $Q_{8}$ in ${ }^{86}$. For $Q_{1}$ the virtual corrections 
at $\mathcal{O}\left(\alpha_{s}\right)$ were calculated in $83,87,88$, but those at $\mathcal{O}\left(\alpha_{s}^{2}\right)$ are known only in the large- $\beta_{0}$ limit ${ }^{89}$. A calculation that goes beyond this approximation by employing an interpolation in the charm quark mass $m_{c}$ was reported in 90 , and has been used in estimating the NNLO branching fraction for the inclusive decay $B \rightarrow X_{s} \gamma^{91}$. Finally, one has to add the Bremsstrahlung contribution $b \rightarrow s \gamma g$ to get the complete decay rate, which in $O\left(\alpha_{s}\right)$ was done in 92 and in $O\left(\alpha_{s}^{2}\right)$ in 93 .

In the $\overline{\mathrm{MS}}$ scheme, the NNLO branching ratio for $E_{\gamma}>1.6 \mathrm{GeV}$ is calculated as 91 .

$$
\mathcal{B}\left(B \rightarrow X_{s} \gamma\right)_{\mathrm{SM}}=(3.15 \pm 0.23) \times 10^{-4}
$$

This amounts to a theoretical precision of about $8 \%$, comparable to the current experimental precision 7

$$
\mathcal{B}\left(B \rightarrow X_{s} \gamma\right)_{\text {Expt. }}=\left(3.55 \pm 0.24_{-0.10}^{+0.09} \pm 0.03\right) \times 10^{-4}
$$

A comparison of the two shows that the SM estimate is in agreement with data though the SM central value lies below the experiment by about $1 \sigma$. This allows for speculations about a beyond-the-SM contribution interfering constructively with the SM amplitude. A case in point is a 2 Higgs doublet model $(2 \mathrm{HDM})$; the preferred value is $m_{H^{+}} \simeq 650 \mathrm{GeV}$ with a $95 \%$ C.L. lower bound $m_{H^{+}}>295 \mathrm{GeV}$. However, more conservatively, the proximity of $\mathcal{B}\left(B \rightarrow X_{s} \gamma\right)$ in the $\mathrm{SM}$ and experiment puts bounds on the parameters characterizing new physics. This has been worked out, together with other constraints, in the context of supersymmetry 94 .

The current (NNLO) theoretical precision on $\mathcal{B}\left(B \rightarrow X_{s} \gamma\right)$ has also been investigated in the context of SCET using a multi-scale OPE involving three low energy scales: $m_{b}, \sqrt{m_{b} \Delta}$ and $\Delta=m_{b}-2 E_{0}$, where $E_{0}$ is the lower cut on the photon energy. Large logarithms associated with these scales are summed at NLL order. The sensitivity to the scale $\Delta$ introduces additional uncertainties. Taking this into account, Becher and Neubert 95 estimate $\mathcal{B}\left(B \rightarrow X_{s} \gamma\right)=(2.98 \pm 0.26) \times 10^{-4}$, which increases the departure of the $\mathrm{SM}$ from data to about $1.4 \sigma$.

\section{2. $B \rightarrow X_{s} \ell^{+} \ell^{-}: S M$ vs. Experiments}

To study the decays $B \rightarrow X_{s} \ell^{+} \ell^{-}$, one has to extend the operator basis in the effective Lagrangian (23) by adding two semileptonic operators 96 :

$$
Q_{9}=\frac{e^{2}}{16 \pi^{2}}\left(\bar{s} \gamma_{\mu} b\right)_{V-A}\left(\bar{\ell} \gamma^{\mu} \ell\right), \quad Q_{10}=\frac{e^{2}}{16 \pi^{2}}\left(\bar{s} \gamma_{\mu} b\right)_{V-A}\left(\bar{\ell} \gamma^{\mu} \gamma_{5} \ell\right)
$$


The corresponding Wilson coefficients $C_{9}(\mu)$ and $C_{10}(\mu)$ have the following perturbative expansion:

$$
\begin{gathered}
C_{9}=\frac{4 \pi}{\alpha_{s}(\mu)} C_{9}^{(-1)}(\mu)+C_{9}^{(0)}(\mu)+\frac{\alpha_{s}(\mu)}{4 \pi} C_{9}^{(1)}(\mu)+\ldots \\
C_{10}=C_{10}^{(0)}+\frac{\alpha_{s}\left(M_{W}\right)}{4 \pi} C_{10}^{(1)}+\ldots .
\end{gathered}
$$

After an expansion in $\alpha_{s}$, the term $C_{9}^{(-1)}(\mu)$ reproduces the dominant part of the electroweak logarithms that originate from photonic penguins with charm quark loops:

$$
\frac{4 \pi}{\alpha_{s}\left(m_{b}\right)} C_{9}^{(-1)}\left(m_{b}\right)=\frac{4}{9} \ln \frac{M_{W}^{2}}{m_{b}^{2}}+O\left(\alpha_{s}\right)
$$

leading to $\frac{4 \pi}{\alpha_{s}\left(m_{b}\right)} C_{9}^{(-1)}\left(m_{b}\right) \simeq 2$. With $C_{9}^{(0)}\left(m_{b}\right) \simeq 2.2$, one needs to calculate in the NNLO accuracy. The NNLO calculation of the decay $B \rightarrow X_{s} l^{+} l^{-}$corresponds to the NLO calculation of $B \rightarrow X_{s} \gamma$, as far as the number of loops in the diagrams is concerned.

The process $B \rightarrow X_{s} \ell^{+} \ell^{-}$differs greatly from the radiative decay $B \rightarrow X_{s} \gamma$ as far as non-perturbative contributions are concerned. The largest effect in $B \rightarrow X_{s} \ell^{+} \ell^{-}$from the intermediate $c \bar{c}$ states comes from the resonances $J / \psi, \psi^{\prime}$ and $\psi^{\prime \prime}$ decaying to $\ell^{+} \ell^{-}$, which can be either modeled, for example, as done by Krüger and Sehgal 97 using dispersion relations and data on $\sigma_{(} e^{+} e^{-} \rightarrow c \bar{c} \rightarrow$ hadrons), or else experimental cuts are imposed on $q^{2}$ to remove the resonant regions and the short-distance contribution is extrapolated through these cuts. Then, there are factorizable $1 / m_{c}$ and $1 / m_{b}$ power corrections, similar to those in $B \rightarrow X_{s} \gamma$, which can be calculated using the OPE and HQET. As is the case for $B \rightarrow X_{s} \gamma$ and $B \rightarrow X_{u} \ell \nu_{\ell}$, there are no $1 / m_{b}$ corrections. The $O\left(1 / m_{b}^{2}\right)$ corrections in this framework were calculated first in 98 and corrected in 99 . The $O\left(1 / m_{b}^{3}\right)$ corrections were calculated in 100 . The $1 / m_{c}$ factorizable power corrections were calculated in 101 .

Including the leading power corrections in $1 / m_{b}$ and $1 / m_{c}$ and taking into account various parametric uncertainties, the branching ratios for the decays $B \rightarrow X_{s} \ell^{+} \ell^{-}$in NNLO are ${ }^{102}$ :

$$
\mathcal{B}\left(B \rightarrow X_{s} e^{+} e^{-}\right)_{\mathrm{SM}} \simeq \mathcal{B}\left(B \rightarrow X_{s} \mu^{+} \mu^{-}\right)_{\mathrm{SM}}=(4.2 \pm 0.7) \times 10^{-6},
$$

where a dilepton invariant mass cut, $m_{\ell \ell}>0.2 \mathrm{GeV}$, has been assumed for comparison with data given below. These estimates make use of the NNLO calculation by Asatryan et al. ${ }^{103}$, restricted to $\hat{s} \equiv q^{2} / m_{b}^{2}<0.25$. The 
spectrum for $\hat{s}>0.25$ has been obtained from the NLO calculations using the scale $\mu_{b} \simeq m_{b} / 2$, as this choice of the scale reduces the NNLO contributions. Subsequent NNLO calculations covered the entire dilepton mass spectrum and are numerically in agreement with this procedure, yielding $\mathcal{B}\left(B \rightarrow X_{s} \mu^{+} \mu^{-}\right)_{\mathrm{SM}}=(4.6 \pm 0.8) \times 10^{-6} 104,105$. The difference in the central values in these results and (30) is of parametric origin.

The BABAR and BELLE collaborations have measured the invariant dilepton and hadron mass spectra in $B \rightarrow X_{s} \ell^{+} \ell^{-}$. Using the SM-based calculations to extrapolate through the cut-regions, the current averages of the branching ratios are ${ }^{7}$.

$$
\begin{aligned}
& \mathcal{B}\left(B \rightarrow X_{s} e^{+} e^{-}\right)=(4.7 \pm 1.3) \times 10^{-6}, \\
& \mathcal{B}\left(B \rightarrow X_{s} \mu^{+} \mu^{-}\right)=\left(4.3_{-1.2}^{+1.3}\right) \times 10^{-6}, \\
& \mathcal{B}\left(B \rightarrow X_{s} \ell^{+} \ell^{-}\right)=\left(4.5_{-1.01}^{+1.03}\right) \times 10^{-6} .
\end{aligned}
$$

Thus, within the current experimental accuracy, which is typically $25 \%$, data and the SM agree with each other in the $b \rightarrow s$ electroweak penguins. The low $q^{2}$-region (say, $q^{2}<8 \mathrm{GeV}^{2}$ ), which allows the most precise comparison with the SM, suffers both from the statistics and a cut on the invariant hadronic mass recoiling against the dilepton. A cut $m_{X}>2 \mathrm{GeV}$ and $m_{X}>1,8 \mathrm{GeV}$ have been used by the BELLE and BABAR collaborations, respectively. The effects of these cuts have been studied in the Fermi-motion model ${ }^{106}$, which has been used in the experimental analysis of the data so far. Subsequently, the $B \rightarrow X_{s} \ell^{+} \ell^{-}$rate with an $m_{X}$ cut in the low- $q^{2}$ region has been calculated using the $B \rightarrow X_{s} \gamma$ shape function 107. This work, whose impact on the analysis of the $B \rightarrow X_{s} \ell^{+} \ell^{-}$ data has yet to be studied, reduces some of the theoretical errors in the SM estimates given in eq. (30). In the same vein, it has also been recently argued 108 that the non-perturbative uncertainties in the large- $q^{2}$ region $\left(q^{2} \geq 14 \mathrm{GeV}^{2}\right)$ can be significantly reduced by normalizing the partial decay width of $B \rightarrow X_{s} \ell^{+} \ell^{-}$with the corresponding partial width of the decay $B \rightarrow X_{u} \ell \nu_{\ell}$. With more data from the $B$ factories, these theoretical developments will enable a more precise test of the SM in the $B \rightarrow X_{s} \ell^{+} \ell^{-}$ decays.

The measurements (27) and (31) provide valuable constraints on beyond-the-SM physics scenarios. Following the earlier analysis to determine the Wilson coefficients in $b \rightarrow s$ transitions $109,102,110$, it has been recently argued ${ }^{111}$ that data now disfavor solutions in which the coefficient $C_{7}^{\text {eff }}$ is similar in magnitude but opposite in sign to the SM coefficient.

Exclusive decays $B \rightarrow\left(K, K^{*}\right) \ell^{+} \ell^{-}\left(\ell^{ \pm}=e^{ \pm}, \mu^{ \pm}\right)$have also been mea- 
sured by the BABAR and BELLE collaborations, and the current world averages of the branching ratios are ${ }^{7}$ :

$$
\begin{gathered}
\mathcal{B}\left(B \rightarrow K \ell^{+} \ell^{-}\right)=(3.9 \pm 0.6) \times 10^{-7}, \\
\mathcal{B}\left(B \rightarrow K^{*} e^{+} e^{-}\right)=\left(11.3_{-2.6}^{+2.8}\right) \times 10^{-7}, \\
\mathcal{B}\left(B \rightarrow K^{*} \mu^{+} \mu^{-}\right)=\left(10.3_{-2.3}^{+2.6}\right) \times 10^{-7}, \\
\mathcal{B}\left(B \rightarrow K^{*} \ell^{+} \ell^{-}\right)=\left(9.4_{-1.6}^{+1.7}\right) \times 10^{-7} .
\end{gathered}
$$

They are also in agreement with the SM-based estimates of the same. A calculation based on the light cone QCD sum rules for the form factors 112 yields ${ }^{102}: \mathcal{B}\left(B \rightarrow K \ell^{+} \ell^{-}\right)=(3.5 \pm 1.2) \times 10^{-7}, \mathcal{B}\left(B \rightarrow K^{*} e^{+} e^{-}\right)=$ $(15.8 \pm 4.9) \times 10^{-7}$, and $\mathcal{B}\left(B \rightarrow K^{*} \mu^{+} \mu^{-}\right)=(11.9 \pm 3.9) \times 10^{-7}$ with the errors dominated by uncertainties on the form factors. In the future, these errors can be reduced by using the data on $B \rightarrow(\pi, \rho) \ell \nu_{\ell}$ to determine the $B \rightarrow(\pi, \rho)$ form factors. This information can be combined with estimates of the $\mathrm{SU}(3)$-symmetry breaking to determine the $B \rightarrow\left(K, K^{*}\right)$ form factors, enabling to predict the FCNC decay rates and spectra more precisely. For the low invariant mass of the dileptons, say $q^{2}<8 \mathrm{GeV}^{2}$, the SCET framework can be employed to reduce the number of form factors and improve the perturbative aspects of these decays.

The Forward-Backward (FB) asymmetry in the decay $B \rightarrow X_{s} \ell^{+} \ell^{-}$, defined as 112

$\overline{\mathcal{A}}_{\mathrm{FB}}\left(q^{2}\right)=\frac{1}{d \mathcal{B}\left(B \rightarrow X_{s} \ell^{+} \ell^{-}\right) / d q^{2}} \int_{-1}^{1} d \cos \theta_{\ell} \frac{d^{2} \mathcal{B}\left(B \rightarrow X_{s} \ell^{+} \ell^{-}\right)}{d q^{2} d \cos \theta_{\ell}} \operatorname{sgn}\left(\cos \theta_{\ell}\right)$,

provides additional constraints on the Wilson coefficients. In particular, the location of the zero-point of this asymmetry (called below $q_{0}^{2}$ ) is a precision tests of the SM. In NNLO, one has the following predictions for the inclusive decays $B \rightarrow X_{s} \ell^{+} \ell^{-}: q_{0}^{2}=(3.90 \pm 0.25) \mathrm{GeV}^{2}\left[\left(3.76 \pm 0.22_{\text {theory }} \pm\right.\right.$ $\left.0.24_{m_{b}}\right) \mathrm{GeV}^{2}$ ], obtained by Ghinculov et al. ${ }^{113}$ [Asatrian et al. ${ }^{114}$ ].

In the SM (and its extensions in which the operator basis remains unchanged), the FB-asymmetry in $B \rightarrow K \ell^{+} \ell^{-}$is zero and in $B \rightarrow$ $K^{*} \ell^{+} \ell^{-}$it depends on the decay form factors. Model-dependent studies yield small form factor-related uncertainties in the zero-point of the asymmetry $\hat{s}_{0}=q_{0}^{2} / m_{B}^{2} 115$. HQET provides a symmetry argument why the uncertainty in $\hat{s}_{0}$ can be expected to be small which is determined by $116 C_{9}^{e f f}\left(\hat{s}_{0}\right)=-\frac{2 m_{b}}{M_{B} \hat{s}_{0}} C_{7}^{e f f}$. However, $O\left(\alpha_{s}\right)$ corrections to the HQETsymmetry relations lead to substantial change in the profile of the FBasymmetry function as well as a significant shift in $\hat{s}_{0} 117,118$. They have been worked out for $B \rightarrow K^{*} \ell^{+} \ell^{-}$using SCET ${ }^{119}$. Restricting ourselves 
to the kinematic region where the light $K^{*}$ meson moves fast and can be viewed approximately as a collinear particle, a factorization formula for the decay amplitude of $B \rightarrow K^{*} \ell^{+} \ell^{-}$, to leading power in $1 / m_{b}$, has been derived in SCET 119 . This coincides formally with the formula obtained earlier by Beneke et al. ${ }^{117}$, using the QCD factorization approach 120, 121 , but is valid to all orders of $\alpha_{s}$ :

$$
\begin{aligned}
\left\langle K_{a}^{*} \ell^{+} \ell^{-}\left|H_{e f f}\right| B\right\rangle & =T_{a}^{I}\left(q^{2}\right) \zeta_{a}\left(q^{2}\right)+ \\
& +\sum_{ \pm} \int_{0}^{\infty} \frac{d \omega}{\omega} \phi_{ \pm}^{B}(\omega) \int_{0}^{1} d u \phi_{K^{*}}^{a}(u) T_{a, \pm}^{I I}\left(\omega, u, q^{2}\right),
\end{aligned}
$$

where $a=\|, \perp$ denotes the polarization of the $K^{*}$ meson. The functions $T_{a}^{I}$ and $T_{a, \pm}^{I I}$ are perturbatively calculable. $\zeta_{a}\left(q^{2}\right)$ are the soft form factors defined in SCET while $\phi_{ \pm}^{B}(\omega)$ and $\phi_{K^{*}}^{a}(u)$ are the light-cone distribution amplitudes (LCDAs) for the $\mathrm{B}$ and $K^{*}$ mesons, respectively. In particular, the location of the zero of the forward-backward asymmetry in $B \rightarrow K^{*} \ell^{+} \ell^{-}$, $q_{0}^{2}$, can be predicted more precisely in SCET due to the improved theoretical precision on the scale dependence of $q_{0}^{2}$.

Including the order $\alpha_{s}$ corrections, the analysis in 119 estimates the zero-point of the FB asymmetry to be

$$
q_{0}^{2}=\left(4.07_{-0.13}^{+0.16}\right) \mathrm{GeV}^{2},
$$

of which the scale-related uncertainty is $\Delta\left(q_{0}^{2}\right)_{\text {scale }}={ }_{-0.05}^{+0.08} \mathrm{GeV}^{2}$ for the range $m_{b} / 2 \leq \mu_{h} \leq 2 m_{b}$ together with the jet function scale $\mu_{l}=$ $\sqrt{\mu_{h} \times 0.5 \mathrm{GeV}}$. This is to be compared with the result given in ${ }^{118}$, also obtained in the absence of $1 / m_{b}$ corrections: $q_{0}^{2}=\left(4.39_{-0.35}^{+0.38}\right) \mathrm{GeV}^{2}$. Of this the largest single uncertainty (about $\pm 0.25 \mathrm{GeV}^{2}$ ) is attributed to the scale dependence. The difference in the estimates of the scale dependence of $q_{0}^{2}$ in 119 and 118 is both due to the incorporation of the SCET logarithmic resummation (done in ${ }^{119}$ ) and the different (scheme-dependent) definitions of the effective form factors for the SCET currents used in these references. Power corrections in $1 / m_{b}$ are probably comparable to the $O\left(\alpha_{s}\right)$ corrections, as argued in 118 . So far, $q_{0}^{2}$ has not been measured experimentally.

BELLE has published the first measurements 5, 122 of the forwardbackward asymmetry (FBA) ${ }^{112}$. The best-fit results by BELLE for the Wilson coefficient ratios for negative value of $C_{7}, \frac{C_{9}}{C_{7}}=-15.3_{-4.8}^{+3.4} \pm 1.1$ and $\frac{C_{10}}{C_{7}}=10.3_{-3.5}^{+5.2} \pm 1.8$, are consistent with the $\mathrm{SM}$ values $C_{9} / C_{7} \simeq-13.7$ and $C_{10} / C_{7} \simeq+14.9$, evaluated in the NLO approximation. However, for the positive value of $C_{7}$, the measurements lead to $\frac{C_{9}}{C_{7}}=-16.3_{-3.7}^{+3.7} \pm 1.1$ and $\frac{C_{10}}{C_{7}}=+11.1_{-3.9}^{+6.0} \pm 1.8$ and the two solutions are of comparable significance. 
With more data at the current $\mathrm{B}$ factories, and yet more anticipated at the LHC, these measurements are expected to become very precise, providing a precision test of the SM in the flavor sector.

\section{3. $B \rightarrow V \gamma: S M$ vs. Experiments}

The decays $B \rightarrow V \gamma\left(V=K^{*}, \rho, \omega\right)$ have been calculated in the NLO approximation using the effective Lagrangian given in (23) and its analogue for $b \rightarrow d$ transitions. Two dynamical approaches, namely the QCD Factorization 120 and pQCD ${ }^{123}$ have been employed to establish factorization of the radiative decay amplitudes in the heavy-quark limit. We illustrate the QCD-F method, where this factorization is worked out for the $B \rightarrow V \gamma 124,125,118,126,127,128,129$ (see 130, 131 for phenomenological updates in NLO, and 132, 133 for the alternative "perturbative QCD" approach). In particular, the matrix element of a given operator in the effective weak Hamiltonian can be written in the form

$$
\left\langle V \gamma\left|Q_{i}\right| \bar{B}\right\rangle=F^{B \rightarrow V_{\perp}} T_{i}^{\mathrm{I}}+\int d \omega d u \phi_{+}^{B}(\omega) \phi_{\perp}^{V}(u) T_{i}^{\mathrm{II}}(\omega, u) .
$$

The non-perturbative effects are contained in $F^{B \rightarrow V_{\perp}}$, the $B \rightarrow V$ transition form factor at $q^{2}=0$, and in $\phi_{+}^{B}$ and $\phi_{\perp}^{V}$, the leading-twist LCDAs of the $B$ - and $V$-mesons. The hard-scattering kernels $T_{i}^{\mathrm{I}}$ and $T_{i}^{\mathrm{II}}$ include only short-distance effects and are calculable in perturbation theory. Contributions to the kernel $T^{\mathrm{I}}$ are closely related to the virtual corrections to the inclusive decay rate, and are referred to as vertex corrections. Those to the kernel $T^{\mathrm{II}}$ are related to parton exchange with the light quark in the $B$-meson, a mechanism commonly referred to as hard spectator scattering. It is expected that the factorization formula is valid up to corrections of $\mathcal{O}\left(\Lambda_{\mathrm{QCD}} / m_{b}\right)$.

The derivation of the factorization formula from a two-step matching procedure in SCET has provided additional insight into its structure. The technical details for $B \rightarrow V \gamma$ in NLO have been provided in 134,135 . In the SCET approach the factorization formula is written as

$$
\left\langle V \gamma\left|Q_{i}\right| \bar{B}\right\rangle=\Delta_{i} C^{A} \zeta_{V_{\perp}}+\frac{\sqrt{m_{B}} F f_{V_{\perp}}}{4} \int d \omega d u \phi_{+}^{B}(\omega) \phi_{\perp}^{V}(u) t_{i}^{\mathrm{II}}(\omega, u),
$$

where $F$ and $f_{V_{\perp}}$ are meson decay constants. The SCET form factor $\zeta_{V_{\perp}}$ is related to the QCD form factor through perturbative and power corrections $117,136,137,138,139,140,141$. In SCET the perturbative hard-scattering kernels are the matching coefficients $\Delta_{i} C^{A}$ and $t_{i}^{\mathrm{II}}$. They are known completely to next-to-leading order $(\mathrm{NLO})\left(\mathcal{O}\left(\alpha_{s}\right)\right)$ in renormalization-group 
(RG) improved perturbation theory 135 . Recently, important steps towards a complete analysis at the next-to-next-to-leading order (NNLO) in $B \rightarrow V \gamma$ decays have been derived in 142 by obtaining full results for the hard-scattering kernels for the dipole operators $Q_{7}$ and $Q_{8}$, and partial results for $Q_{1}$, valid in the large- $\beta_{0}$ limit and neglecting NNLO corrections from spectator scattering. In addition, this work provides the virtual corrections to this order for the $B \rightarrow V \gamma$ decays, as they can not be obtained from the published calculations for the inclusive decay $B \rightarrow X_{s} \gamma$, discussed previously.

In SCET the hard-scattering kernel $t_{i}^{\mathrm{II}}$ for a given operator is subfactorized into the convolution of a hard-coefficient function with a universal jet function, in the form

$$
t_{i}^{\mathrm{II}}(u, \omega)=\int_{0}^{1} d \tau \Delta_{i} C^{B 1}(\tau) j_{\perp}(\tau, u, \omega) \equiv \Delta_{i} C^{B 1} \star j_{\perp} .
$$

The hard coefficients $\Delta_{i} C^{B 1}$ contain physics at the hard scale $m_{b}$, while the jet function $j_{\perp}$ contains physics at the hard-collinear scale $\sqrt{m_{b} \Lambda}$. The hard coefficient is identified in a first step of matching QCD $\rightarrow \mathrm{SCET}_{\mathrm{I}}$, and the jet function in a second step of matching $\mathrm{SCET}_{\mathrm{I}} \rightarrow \mathrm{SCET}_{\mathrm{II}}$. Details in NLO have been worked out for $B \rightarrow V \gamma$ in 134,135 .

The effective field-theory techniques are crucial for providing a fieldtheoretical definition of the objects in (37), and for resumming large perturbative logarithms of the ratio $m_{b} / \Lambda_{\mathrm{QCD}}$ in $t_{i}^{\mathrm{II}}$. In the effective-theory approach resummation is carried out by solving the renormalization-group equations for the matching coefficients $\Delta_{i} C^{B 1}$. Since these coefficients enter the factorization formula in a convolution with the jet function $j_{\perp}$, their anomalous dimension is a distribution in the variables $\tau$ and $u$. The evolution equations must be solved before performing the convolution with $j_{\perp}$. Therefore, resummation is not possible in the original QCD factorization formula (36), where the hard-scattering kernels $T_{i}^{\mathrm{II}}$ are obtained only after this convolution has been carried out.

Using the SCET framework, the branching ratios in the NNLO are as follows 142 :

$$
\begin{aligned}
\mathcal{B}_{\mathrm{NNLO}}\left(B^{0} \rightarrow K^{* 0} \gamma\right) & \simeq(4.3 \pm 1.4) \times 10^{-5} \\
\mathcal{B}_{\mathrm{NNLO}}\left(B^{ \pm} \rightarrow K^{* \pm} \gamma\right) & \simeq(4.6 \pm 1.4) \times 10^{-5} \\
\mathcal{B}_{\mathrm{NNLO}}\left(B_{s}^{0} \rightarrow \phi \gamma\right) & \simeq(4.3 \pm 1.4) \times 10^{-5}
\end{aligned}
$$

It should be noted that, very much like the $B \rightarrow X_{s} \gamma$ case, the complete NNLO calculations for the virtual corrections to the matrix element of 
the operator $O_{1}$ in $B \rightarrow K^{*} \gamma$ are not yet at hand. In addition, the hard spectator corrections from this operator are calculated only in NLO. In the NNLO branching ratios quoted above, the errors are increased to take these missing pieces into account.

The above theoretical branching ratios, compared with the current experimental measurements 6,7 , yield the following results 142 :

$$
\begin{gathered}
\frac{\mathcal{B}_{\mathrm{NNLO}}\left(B^{0} \rightarrow K^{* 0} \gamma\right)}{\mathcal{B}_{\mathrm{Expt}}\left(B^{0} \rightarrow K^{* 0} \gamma\right)}=1.1 \pm 0.35 \pm 0.06, \\
\frac{\mathcal{B}_{\mathrm{NNLO}}\left(B^{ \pm} \rightarrow K^{* \pm} \gamma\right)}{\mathcal{B}_{\mathrm{Expt}}\left(B^{ \pm} \rightarrow K^{* \pm} \gamma\right)}=1.1 \pm 0.35 \pm 0.07, \\
\frac{\mathcal{B}_{\mathrm{NNLO}}\left(B_{s}^{0} \rightarrow \phi \gamma\right)}{\mathcal{B}_{\mathrm{Expt}}\left(B_{s}^{0} \rightarrow \phi \gamma\right)}=0.8 \pm 0.2 \pm 0.3
\end{gathered}
$$

The decays $B \rightarrow(\rho, \omega) \gamma$ involve in addition to the (short-distance) penguin amplitude also significant long-distance contributions, in particular in the decays $B^{ \pm} \rightarrow \rho^{ \pm} \gamma$. In the factorization approximation, typical Annihilation-to-Penguin amplitude ratio is estimated as ${ }^{143}: \epsilon_{\mathrm{A}}\left(\rho^{ \pm} \gamma\right)=$ $0.30 \pm 0.07 . O\left(\alpha_{s}\right)$ corrections to the annihilation amplitude in $B \rightarrow \rho \gamma$ are not known; also the proof of factorization of this amplitude is still not at hand. The annihilation contribution to the decays $B^{0} \rightarrow \rho^{0} \gamma$ and $B^{0} \rightarrow \omega \gamma$ is expected to be suppressed (relative to the corresponding amplitude in $\left.B^{ \pm} \rightarrow \rho^{ \pm} \gamma\right)$ due to the electric charges $\left(Q_{d} / Q_{u}=-1 / 2\right)$ and the color factors, and the corresponding $A / P$ ratio for these decays is estimated as $\epsilon_{\mathrm{A}}\left(\rho^{0} \gamma\right) \simeq-\epsilon_{\mathrm{A}}(\omega \gamma) \simeq 0.05$

The decay amplitudes for $B \rightarrow(\rho, \omega) \gamma$ depend on the CKM matrix elements $V_{t d}^{*} V_{t b}$ (from the penguin diagrams) and $V_{u b}^{*} V_{u d}$ (from the annihilation diagrams). Hence, these decays provide potentially very powerful constraints on the CKM parameters, $\bar{\rho}$ and $\bar{\eta}$. Since a large number of observables can be measured in these decays, such as the individual branching ratios for $B^{ \pm} \rightarrow \rho^{ \pm} \gamma$ and $B^{0} \rightarrow\left(\rho^{0}, \omega\right) \gamma$, isospin- and $\mathrm{SU}(3)$ violating asymmetries in the decay rates, and direct and time-dependent $\mathrm{CP}$ asymmetries, they have been studied theoretically in a number of papers $124,125,127,128,130,131,144$. Experimentally, a beginning has been made in the measurements of the $b \rightarrow d \gamma$ transition through the measurements of the branching ratios for $B \rightarrow(\rho, \omega) \gamma$, reported by BABAR and BELLE. Current measurements are not very precise, as can be seen from the current world averages ${ }^{7}$ (in units of $10^{-6}$ ): $\mathcal{B}\left(B^{ \pm} \rightarrow \rho^{ \pm} \gamma\right)=0.96 \pm 0.23$, $\mathcal{B}\left(B^{0} \rightarrow \rho^{0} \gamma\right)=0.77 \pm 0.14$ and $\mathcal{B}\left(B^{0} \rightarrow \omega \gamma\right)=0.41 \pm 0.15$. In addition, theoretical estimates suffer from large hadronic uncertainties, dominated 
by the imprecise knowledge of the form factors. Hence, the resulting constraints on the CKM parameters are not very quantitative. Theoretical uncertainties are greatly reduced in the ratios of the branching ratios involving the decays $B \rightarrow(\rho, \omega) \gamma$ and $B \rightarrow K^{*} \gamma$. Calling the ratios of the branching ratios $R^{ \pm}\left(\rho \gamma / K^{*} \gamma\right)$ and $R^{0}\left(\rho \gamma / K^{*} \gamma\right)$, for the decays of the $B^{ \pm}$ and $B^{0}$ mesons, respectively, one has 124

$$
\begin{aligned}
& R^{ \pm}\left(\rho \gamma / K^{*} \gamma\right)=\left|\frac{V_{t d}}{V_{t s}}\right|^{2} \frac{\left(M_{B}^{2}-M_{\rho}^{2}\right)^{3}}{\left(M_{B}^{2}-M_{K^{*}}^{2}\right)^{3}} \zeta^{2}\left(1+\Delta R^{ \pm}\left(\epsilon_{\mathrm{A}}^{ \pm}, \bar{\rho}, \bar{\eta}\right)\right), \\
& R^{0}\left(\rho \gamma / K^{*} \gamma\right)=\frac{1}{2}\left|\frac{V_{t d}}{V_{t s}}\right|^{2} \frac{\left(M_{B}^{2}-M_{\rho}^{2}\right)^{3}}{\left(M_{B}^{2}-M_{K^{*}}^{2}\right)^{3}} \zeta^{2}\left(1+\Delta R^{0}\left(\epsilon_{\mathrm{A}}^{0}, \bar{\rho}, \bar{\eta}\right)\right),
\end{aligned}
$$

where $\zeta=T_{1}^{\rho}(0) / T_{1}^{K^{*}}(0)$, with $T_{1}^{\rho}(0)$ and $T_{1}^{K^{*}}(0)$ being the transition form factors evaluated at $q^{2}=0$ in the decays $B \rightarrow \rho \gamma$ and $B \rightarrow K^{*} \gamma$, respectively. The functions $\Delta R^{ \pm}\left(\epsilon_{\mathrm{A}}^{ \pm}, \bar{\rho}, \bar{\eta}\right)$ and $\Delta R^{0}\left(\epsilon_{\mathrm{A}}^{0}, \bar{\rho}, \bar{\eta}\right)$, appearing on the r.h.s. of the above equations encode both the $O\left(\alpha_{s}\right)$ contribution to the penguin amplitudes and annihilation contributions, with the latter estimated so far only in the lowest order. They have a non-trivial dependence on the CKM parameters $\bar{\rho}$ and $\bar{\eta} 124,125$. Theoretical uncertainty in the evaluation of the ratios $R^{ \pm}\left(\rho \gamma / K^{*} \gamma\right)$ and $R^{0}\left(\rho \gamma / K^{*} \gamma\right)$ is dominated by the error on the quantity $\zeta$, and to some extent also by the errors on the parameters $\epsilon_{A}^{ \pm}$and $\epsilon_{A}^{0}$, characterizing the annihilation/penguin ratios. In the $\mathrm{SU}(3)$ limit $\zeta=1$; $\mathrm{SU}(3)$-breaking corrections have been calculated in several approaches, including the QCD sum rules and Lattice QCD. With the current values for the ratios $R^{ \pm}\left(\rho \gamma / K^{*} \gamma\right)=0.032 \pm 0.008$, $R^{0}\left(\rho \gamma / K^{*} \gamma\right)=0.039 \pm 0.007$ and $R^{0}\left(\omega \gamma / K^{*} \gamma\right)=0.021 \pm 0.007$, the current world average of $\left|V_{t d} / V_{t s}\right|$ from the ratio of $B \rightarrow(\rho, \omega) \gamma$ and $B \rightarrow K^{*} \gamma$ is 145 :

$$
\left|V_{t d} / V_{t s}\right|=0.194_{-0.014}^{+0.015}(\exp ) \pm 0.014(\mathrm{th})
$$

where the Light-cone QCD sum rules 131 have been used to estimate the hadronic input quantities. This determination is compatible with the one from the mass difference ratio $\Delta M_{B_{s}} / \Delta M_{B_{d}}$ given in eq. (22), but less precise.

\section{5. $B \rightarrow M_{1} M_{2}$ Decays}

Exclusive non-leptonic decays are the hardest nuts to crack in the theory of $B$-decays. Basically, there are four different theoretical approaches to calculate and/or parameterize the hadronic matrix elements in $B \rightarrow M_{1} M_{2}$ decays: 
(1) $\mathrm{SU}(2) / \mathrm{SU}(3)$ symmetries and phenomenological Ansaetze $146,147,148,149$

(2) Dynamical approaches based on perturbative QCD, such as the QCD Factorization 120 and the competing pQCD approach 123 .

(3) Charming Penguins 150 using the renormalization group invariant topological approach of Buras and Silvestrini 151.

(4) Soft Collinear Effective Theory (SCET) 43, 44, 45, 46, which we have already discussed in the context of radiative and semileptonic decays.

These approaches will be discussed on the example of the $B \rightarrow \pi \pi$ and $B \rightarrow K \pi$ decays for which now there exist enough data to extract the underlying dynamical parameters. Prior to this, however, we discuss the measurements of the angle $\beta$ (or $\phi_{1}$ ) from the experiments at the $B$ factories.

\subsection{Interplay of Mixing and Decays of $B^{0}$ - and $\bar{B}^{0}$-Mesons to CP Eigenstates}

We start with the discussion of the transition $b \rightarrow c c \bar{s}$, which is dominated by the tree topology. The time-dependent $\mathrm{CP}$ asymmetry in the decays $B^{0} \rightarrow f$ and $\bar{B}^{0} \rightarrow f$, where $f$ is a CP eigenstate, such as $J / \psi K_{s}$ and $J / \psi K_{L}$, is defined as:

$$
\mathcal{A}_{f}(t)=\frac{\Gamma\left[\bar{B}^{0}(t) \rightarrow f\right]-\Gamma\left[B^{0}(t) \rightarrow f\right]}{\Gamma\left[\bar{B}^{0}(t) \rightarrow f\right]+\Gamma\left[B^{0}(t) \rightarrow f\right]} .
$$

The time evolution of the two flavor eigenstates $B^{0}$ and $\bar{B}^{0}$ is determined by $(2 \times 2)$ Hermitian matrices $M$ and $\Gamma$. The physical states (with definite masses and lifetimes) are the linear combinations of $B^{0}$ and $\bar{B}^{0}$, with $\left|B_{d}(L, H)\right\rangle=p\left|B^{0}\right\rangle \pm q\left|\bar{B}^{0}\right\rangle$, dependent on two complex parameters $p$ and $q$. Defining the decay amplitudes $A(f) \equiv\left\langle f|H| B^{0}\right\rangle$ and $\bar{A}(f) \equiv\left\langle f|H| \bar{B}^{0}\right\rangle$ of the $B^{0}$ - and $\bar{B}^{0}$-mesons into the final state $f$, the time-dependent CP asymmetry is determined by the quantity $\lambda_{f}$, involving the interplay of mixing and decay amplitudes:

$$
\lambda_{f}=\frac{q}{p} \rho(f), \quad \rho(f)=\frac{\bar{A}(f)}{A(f)} .
$$

For the $B_{d}^{0}-\bar{B}_{d}^{0}$ mixing, the ratio $q / p$ involves the phase $\beta$ (or $\phi_{1}$ ), which is one of the angles of the unitarity triangle:

$$
\frac{q}{p}=\frac{V_{t b}^{*} V_{t d}}{V_{t b} V_{t d}^{*}}=\mathrm{e}^{-2 i \phi_{\text {mixing }}}=\mathrm{e}^{-2 i \beta} .
$$


The time dependent CP asymmetry (41) is then expressed as

$$
\mathcal{A}_{f}(t)=C_{f} \cos \left(\Delta M_{B_{d}} t\right)+S_{f} \sin \left(\Delta M_{B_{d}} t\right),
$$

where $\Delta M_{B_{d}}=(0.507 \pm 0.005) \mathrm{ps}^{-1}$ is the mass difference between the heavy and light $B_{d}^{0}$-meson mass eigenstates and the difference in the decay widths $\Delta \Gamma_{B_{d}}$ has been neglected. The quantities $C_{f}$ and $S_{f}$, called the direct and mixing-induced $\mathrm{CP}$ asymmetries, respectively, are defined in terms of the complex variable $\lambda_{f}$ as follows:

$$
C_{f}=\frac{1-\left|\lambda_{f}\right|^{2}}{1+\left|\lambda_{f}\right|^{2}}, \quad S_{f}=\frac{2 \operatorname{Im} \lambda_{f}}{1+\left|\lambda_{f}\right|^{2}} .
$$

If the decays $B^{0} \rightarrow f$ and $\bar{B}^{0} \rightarrow f$ are dominated by a single amplitude, the ratio $\rho(f)=\eta_{f} \mathrm{e}^{-2 i \phi_{\text {decay }}}$, where $\eta_{f}= \pm 1$ is the CP parity of the state $f$, is a pure phase factor and the asymmetries (45) reduce to the expressions:

$$
C_{f}=0, \quad S_{f}=-\eta_{f} \sin 2\left(\phi_{\text {mixing }}+\phi_{\text {decay }}\right) .
$$

The decays $B^{0} / \bar{B}^{0} \rightarrow J / \psi K_{s}, J / \psi K_{L}$, and a number of related final states with $f$ being $\psi(2 S) K_{s}, \eta_{c} K_{s}, \chi_{c 1} K_{s}$, and $J / \psi K^{* 0}\left(K^{* 0} \rightarrow K_{s} \pi^{0}\right)$ belong to the category of gold plated decays 152 . In all these modes, the direct CP asymmetry $C_{f}$, to a very high accuracy, vanishes, and the quantity $S_{f}$, the mixing-induced $\mathrm{CP}$ asymmetry, measures $\sin (2 \beta)$. Averaging over all the decay channels, the results of the BABAR and BELLE measurements are as follows 4 :

$$
\begin{array}{lll}
C=0.049 \pm 0.022 \pm 0.017, & S=0.714 \pm 0.032 \pm 0.018, & {[\mathrm{BABAR}]} \\
C=-0.019 \pm 0.025, & S=0.651 \pm 0.034 . & \text { [BELLE] }
\end{array}
$$

In the BABAR result, the first error is statistical and the second is systematic, while in the BELLE data both the errors have been combined. The current world average for $S_{f}=\sin (2 \beta)$ for the quark transition $b \rightarrow c \bar{c} s$ is 7 :

$$
\sin (2 \beta)=0.681 \pm 0.025
$$

where the data from LEP and Tevatron have also been included. Restricting $\beta$ in the range $0 \leq \beta \leq \pi / 2$, two possible values can be extracted $\beta=(21.5 \pm$ $1.0)^{\circ}$ and $\beta=(68.5 \pm 1.0)^{\circ}$. The two-fold ambiguity has now been resolved by several $\cos (2 \beta)$ measurements, involving the Dalitz analysis of the decay modes $B^{0} \rightarrow D_{3 \text {-body }}^{0} h^{0}, B^{0} \rightarrow K_{s} \pi^{+} \pi^{-}, B^{0} \rightarrow K_{s} K^{+} K^{-}$, and the older results on $B^{0} \rightarrow J / \psi K^{* 0}$, leading to the determination $\beta=(21.5 \pm 1.0)^{\circ}$.

The direct measurement of $\sin (2 \beta)$ in eq.(49) is to be compared with the indirect estimate of the same, obtained from the fits of the CKM unitarity 
triangle (UT). For this, the UTfit collaboration 66 quotes $\sin (2 \beta)=0.739 \pm$ 0.044 , obtained from the sides of the UT alone, and $\sin (2 \beta)=0.736 \pm$ 0.042 , by including also the CP-violating quantity $\epsilon_{K}$ in the $K$-decays. The results from the CKMfitter group 67 are similar. Thus, SM passes this test comfortably.

Another key test of the SM in the flavor sector is to compare the CP-violating quantities $S_{f}$ and $C_{f}$ involving the penguin-topology dominated quark transitions $b \rightarrow s \bar{s} s$ and $b \rightarrow s d \bar{d}$ with the ones from the transition $b \rightarrow c \bar{c} s$, dominated by the tree topology and discussed quantitatively above. The point here is that penguin amplitudes may receive contributions from New Physics. For example, new phases, present generically in supersymmetric theories, may reveal themselves, leading to

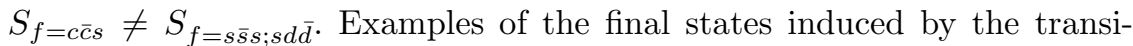
tion $b \rightarrow s \bar{s} s$ are $\left(\phi, \eta, \eta^{\prime}, K \bar{K}\right) K_{s}$, and the ones induced by the transition $b \rightarrow s d \bar{d}$ are $K_{s}^{0}\left(\pi^{0}, \rho^{0}, \omega\right)\left(B^{0} \rightarrow \eta^{\prime} K_{s}\right.$ receives contributions from both the transitions). The current measurements of $S_{f}=-\eta_{f} \sin \left(2 \beta^{\mathrm{eff}}\right)$ from the penguin-dominated decays are ${ }^{7}: S_{\phi K^{0}}=0.39 \pm 0.17, S_{\eta^{\prime} K^{0}}=$ $0.61 \pm 0.07, S_{K_{s} K_{s} K_{s}}=0.58 \pm 0.20, S_{\pi^{0} K_{s}}=0.38 \pm 0.19, S_{\rho^{0} K_{s}}=0.61_{-0.27}^{+0.25}$, $S_{\omega K_{s}}=0.48 \pm 0.24, S_{f_{0} K^{0}}=0.84 \pm 0.07, S_{K^{+} K^{-} K^{0}}=0.73 \pm 0.10$, and $S_{\pi^{0} \pi^{0} K_{s}}=-0.52 \pm 0.41$. These measurements are not as precise as the ones from the $b \rightarrow c \bar{c} s$ decays due to the much smaller branching ratios (typically $10^{-5}$ ) compared to the decay $B^{0} \rightarrow J / \psi K_{s}$. Also, they involve more than one decay topologies. However, within (large) errors, the values of $\sin \left(2 \beta^{\text {eff }}\right)$ from the penguin-dominated transitions are consistent with the value of $\sin (2 \beta)$ from the tree-dominated transition given above in eq.(49), with the possible exception of $S_{\phi K^{0}}$, which deviates by about $2 \sigma$, and the poorly measured odd-man out $S_{\pi^{0} \pi^{0} K_{s}}$. It seems that the fog on $S_{f}$ in the penguindominated decays from the initial epoch of the $B$-factory experiments has largely evaporated, and the emerging contours of $\mathrm{CP}$ asymmetries in these decays are very much the same as predicted by the SM.

\section{2. $B \rightarrow \pi \pi: S M$ vs. Experiments}

The determination of the phase $\alpha$ is based on the branching ratios and $\mathrm{CP}$ asymmetries in the quark transition $b \rightarrow u \bar{u} d$. They metamorphise in the decays $B \rightarrow \pi \pi, B \rightarrow \rho \pi$ and $B \rightarrow \rho \rho$, apart from other final states. We concentrate here on the decay $B \rightarrow \pi \pi$, which has received a lot of theoretical attention.

There are three dominant topologies in the $B \rightarrow \pi \pi$ decays termed as Tree $(\mathrm{T})$, Penguin $(\mathrm{P})$ and Color-suppressed $(\mathrm{C})$. In addition, there are 
several other subdominant topologies which will be neglected in the discussion below. Parametrization of the T, P, and C amplitudes is conventiondependent. In the Gronau-Rosner c-convention ${ }^{149}$, these amplitudes can be represented as

$$
\begin{aligned}
\sqrt{2} A^{+0} & =-|T| \mathrm{e}^{i \delta_{T}} \mathrm{e}^{i \gamma}\left[1+|C / T| \mathrm{e}^{i \Delta}\right], \\
A^{+-} & =-|T| \mathrm{e}^{i \delta_{T}}\left[\mathrm{e}^{i \gamma}+|P / T| \mathrm{e}^{i \delta}\right], \\
\sqrt{2} A^{00} & =-|T| \mathrm{e}^{i \delta_{T}}\left[|C / T| \mathrm{e}^{i \Delta} \mathrm{e}^{i \gamma}-|P / T| \mathrm{e}^{i \delta}\right] .
\end{aligned}
$$

The charged-conjugate amplitudes $\bar{A}^{i j}$ differ by the replacement $\gamma \rightarrow-\gamma$. The amplitudes (50) and the charged-conjugate ones obey the isospin relations:

$$
A^{+0}=\frac{1}{\sqrt{2}} A^{+-}+A^{00}, \quad \bar{A}^{-0}=\frac{1}{\sqrt{2}} \bar{A}^{+-}+\bar{A}^{00} .
$$

There are 5 dynamical parameters $|T|, r \equiv|P / T|, \delta,|C / T|, \Delta$, with $\delta_{T}=0$ assumed for the overall phase. Thus, the weak phase $\gamma$ can be extracted together with other quantities if the complete set of experimental data on $B \rightarrow \pi \pi$ decays is available.

Table 1. Branching ratios (in units of $10^{-6}$ ) and CP asymmetries in the $B \rightarrow \pi \pi$ decays

$$
\begin{array}{ll}
\mathcal{B}\left(B^{+} \rightarrow \pi^{+} \pi^{0}\right)=5.59_{-0.40}^{+0.41} & A_{\mathrm{CP}}\left(\pi^{+} \pi^{0}\right)=0.06 \pm 0.05 \\
\mathcal{B}\left(B^{0} \rightarrow \pi^{0} \pi^{0}\right)=1.31 \pm 0.21 & A_{\mathrm{CP}}\left(\pi^{0} \pi^{0}\right)=0.48_{-0.31}^{+0.32} \\
\mathcal{B}\left(B^{0} \rightarrow \pi^{+} \pi^{-}\right)=5.16 \pm 0.22 & C_{\mathrm{CP}}\left(\pi^{+} \pi^{-}\right)=-0.38 \pm 0.07 \\
& S_{\mathrm{CP}}\left(\pi^{+} \pi^{-}\right)=-0.61 \pm 0.08
\end{array}
$$

The experimental branching ratios and the direct $\mathrm{CP}$ asymmetries $A_{\mathrm{CP}}\left(\pi^{0} \pi^{0}\right)$ and $C_{\mathrm{CP}}\left(\pi^{+} \pi^{-}\right)$, as well as the value of the coefficient $S_{\mathrm{CP}}\left(\pi^{+} \pi^{-}\right)$in time-dependent CP asymmetry, presented in Table 1 , have been fitted to determine the various parameters (the direct $\mathrm{CP}$ asymmetry $A_{\mathrm{CP}}\left(\pi^{+} \pi^{0}\right)$ is not relevant for this analysis but can be important in determining the size of electroweak contribution in the decays considered). An updated analysis by Parkhomenko based on the paper ${ }^{153}$ yields the following values for the hadronic parameters:

$$
\begin{array}{ll}
|P / T|=0.473_{-0.055}^{+0.060}, & \delta=\left(-40.2_{-4.7}^{+6.8}\right)^{\circ}, \\
|C / T|=0.966_{-0.061}^{+0.058}, & \Delta=\left(-56.3_{-7.9}^{+8.4}\right)^{\circ},
\end{array}
$$


and for the CKM unitarity triangle angle $\gamma$ (or equivalently $\alpha$ )

$$
\gamma=\left(65.9_{-3.2}^{+3.0}\right)^{\circ}, \quad \alpha=\pi-\beta-\gamma=\left(92.6_{-3.2}^{+3.4}\right)^{\circ} .
$$

Similar fits based on their data have been performed by the BABAR and BELLE collaborations resulting in slightly larger values: $\alpha=\left(96_{-6}^{+10}\right)^{\circ}$ (BABAR) and $\alpha=(97 \pm 11)^{\circ}$ (BELLE). The overall fits performed by the CKM-Fitter and UT-Fit groups prefer slightly smaller values, yielding: $\alpha=\left(90.7_{-2.9}^{+4.5}\right)^{\circ} \quad 67$ and $\alpha=(88.7 \pm 6.2)^{\circ} 66$, respectively. All the above estimates are in good agreement with each other within the quoted errors, stating that the data on $B \rightarrow \pi \pi$ (as well as the other decay modes $B \rightarrow \rho \pi$ and $B \rightarrow \rho \rho)$ are in agreement with the indirect estimate of the phase $\alpha$ from the unitarity triangle. The strong phases $\delta$ and $\Delta$ in Eq.(52) come out rather large. In particular, they are much larger than the predictions of the QCD-F approach ${ }^{120}$, with pQCD 123 in better agreement with data, but neither of these approaches provides a good fit of the entire $B \rightarrow \pi \pi$ data.

Data on $B \rightarrow \pi \pi$ decays are in agreement with the phenomenological approach of the so-called charming penguins ${ }^{154}$, and with the SCET-based analyses by Bauer et al. 155, 156 which also attributes a dominant role to the charming penguin amplitude. However, a proof of the factorization of the charming penguin amplitude in the SCET approach remains to be provided. In addition, SCET makes a number of predictions in the $B \rightarrow \pi \pi$ sector, such as the branching ratio $\mathcal{B}\left(B^{0} \rightarrow \pi^{0} \pi^{0}\right)$ : 155

$$
\left.\mathcal{B}\left(B^{0} \rightarrow \pi^{0} \pi^{0}\right)\right|_{\gamma=64^{\circ}}=(1.3 \pm 0.6) \times 10^{-6} .
$$

In contrast, predictions of the QCD-F and pQCD approaches are rather similar: $\mathcal{B}\left(B^{0} \rightarrow \pi^{0} \pi^{0}\right) \sim 0.3 \times 10^{-6}$, in substantial disagreement with the data.

\subsection{Present bounds on the phase $\gamma$ from $B$ decays}

The classic method for determining the phase $\gamma$ (or $\left.\phi_{3}\right) 157,158,159,160$ involves the interference of the tree amplitudes $b \rightarrow u W^{-} \rightarrow u \bar{c} s$ leading to $B^{-} \rightarrow D^{0} K^{-}$and $b \rightarrow c W^{-} \rightarrow c \bar{u} s$ leading to $B^{-} \rightarrow \overline{D^{0}} K^{-}$. These amplitudes can interfere if $D^{0}$ and $\overline{D^{0}}$ decay into a common hadronic final state. Noting that the $\mathrm{CP}= \pm 1$ eigenstates $D_{ \pm}^{0}$ are linear combinations of the $D^{0}$ and $\overline{D^{0}}$ states: $D_{ \pm}^{0}=\left(D^{0} \pm \overline{D^{0}}\right) / \sqrt{2}$, both branches lead to the same final states $B^{-} \rightarrow D_{ \pm}^{0} K^{-}$. So, the condition of CP interferometry is fulfilled. The decays $B^{-} \rightarrow D_{ \pm}^{0} K^{-}$are described by the amplitudes:

$$
A\left(B^{-} \rightarrow D_{ \pm}^{0} K^{-}\right)=\frac{1}{\sqrt{2}}\left[A\left(B^{-} \rightarrow D^{0} K^{-}\right) \pm A\left(B^{-} \rightarrow \overline{D^{0}} K^{-}\right)\right] .
$$


Since, the weak phase of the $b \rightarrow u$ transition is $\gamma$ but the $b \rightarrow c$ transition has no phase, a measurement of the CP asymmetry through the interference of these two amplitudes yields $\gamma$. The four equations that will be used to extract $\gamma$ are:

$$
\begin{aligned}
& R_{ \pm} \equiv \frac{\mathcal{B}\left(B^{-} \rightarrow D_{ \pm}^{0} K^{-}\right)+\mathcal{B}\left(B^{+} \rightarrow D_{ \pm}^{0} K^{+}\right)}{\mathcal{B}\left(B^{-} \rightarrow D^{0} K^{-}\right)+\mathcal{B}\left(B^{+} \rightarrow D^{0} K^{+}\right)}=1+r_{\mathrm{DK}}^{2} \pm 2 r_{\mathrm{DK}} \cos \delta_{\mathrm{DK}} \cos \gamma \\
& A_{ \pm} \equiv \frac{\mathcal{B}\left(B^{-} \rightarrow D_{ \pm}^{0} K^{-}\right)-\mathcal{B}\left(B^{+} \rightarrow D_{ \pm}^{0} K^{+}\right)}{\mathcal{B}\left(B^{-} \rightarrow D_{ \pm}^{0} K^{-}\right)+\mathcal{B}\left(B^{+} \rightarrow D_{ \pm}^{0} K^{+}\right)}=\frac{ \pm 2 r_{\mathrm{DK}} \sin \delta_{\mathrm{DK}} \sin \gamma}{1+r_{\mathrm{DK}}^{2} \pm 2 r_{\mathrm{DK}} \cos \delta_{\mathrm{DK}} \cos \gamma}
\end{aligned}
$$

Here, $r_{\mathrm{DK}}$ is the ratio of the two tree amplitudes $161 r_{\mathrm{DK}} \equiv\left|T_{1} / T_{2}\right| \sim$ $(0.1-0.2)$, with $T_{1}$ and $T_{2}$ being the CKM suppressed $(b \rightarrow u)$ and CKM allowed $(b \rightarrow c)$ amplitudes, respectively, and $\delta_{\mathrm{DK}}$ is the relative strong phase between them. The construction of the final states involves flavor and CP-tagging of the various $D^{0}$ states, which can be done, for example, through the decays $D_{+}^{0} \rightarrow \pi^{+} \pi^{-}, D_{-}^{0} \rightarrow K_{S} \pi^{0}$, and $D^{0} \rightarrow K^{-} \pi^{+}$. With three unknowns $\left(r_{\mathrm{DK}}, \delta_{\mathrm{DK}}, \gamma\right)$, but four quantities which will be measured, $R_{ \pm}$and $A_{ \pm}$, one has, in principle, an over constrained system.

Experimentally, the quantities $R_{ \pm}$are measured through the ratios:

$$
R(K / \pi) \equiv \frac{\mathcal{B}\left(B^{-} \rightarrow D^{0} K^{-}\right)}{\mathcal{B}\left(B^{-} \rightarrow D^{0} \pi^{-}\right)}, \quad R(K / \pi)_{ \pm} \equiv \frac{\mathcal{B}\left(B^{ \pm} \rightarrow D_{ \pm}^{0} K^{ \pm}\right)}{\mathcal{B}\left(B^{ \pm} \rightarrow D_{ \pm}^{0} \pi^{ \pm}\right)}
$$

With all three quantities $R(K / \pi)$ and $R(K / \pi)_{ \pm}$measured, one can determine $R_{ \pm}=R(K / \pi)_{ \pm} / R(K / \pi)$. More useful decay modes to construct the $B \rightarrow D K$ triangle can be added to reduce the statistical errors. Along these lines, Atwood and Soni ${ }^{162}$ have advocated to also include the decays of the vector states in the analysis, such as $B^{-} \rightarrow K^{*-} D^{0}, B^{-} \rightarrow K^{-} D^{* 0}$, and $B^{-} \rightarrow K^{*-} D^{* 0}$, making use of the $D^{* 0} \rightarrow D^{0} \gamma$ and $D^{* 0} \rightarrow D^{0} \pi^{0}$ modes.

Present measurements in the $B \rightarrow D K$ and $B \rightarrow D \pi$ decays by the BABAR and BELLE collaborations yielding $R_{ \pm}$and $A_{ \pm}$for the $D_{\mathrm{CP}} K^{-}$ mode are summarized by HFAG ${ }^{7}$ :

$$
\begin{array}{ll}
R_{+}=1.09 \pm 0.09, & A_{+}=0.26 \pm 0.08 \quad[\mathrm{BELLE}, \mathrm{BABAR}] \\
R_{-}=0.90 \pm 0.10, & A_{-}=-0.16 \pm 0.09 \quad[\mathrm{BELLE}]
\end{array}
$$

The corresponding quantities for the $D_{\mathrm{CP}}^{*} K^{-}$and $D_{\mathrm{CP}} K^{*-}$ are also given by $\mathrm{HFAG}^{7}$.

A modification of the Gronau-London-Wyler (GLW) method described above has been suggested by Atwood, Dunietz and Soni (ADS), where $B^{-} \rightarrow D^{0} K^{-}$with $D^{0} \rightarrow K^{+} \pi^{-}$(or similar) and the charge conjugate 
decays are implied. BABAR and BELLE use the following definitions for the quantities called $A_{\mathrm{ADS}}$ and $R_{\mathrm{ADS}}$, (the decay modes $B^{-} \rightarrow D^{0} K^{-}$ followed by $D^{0} \rightarrow K^{+} \pi^{-}$are used to exemplify the method)

$$
\begin{aligned}
R_{\mathrm{ADS}} & \equiv \frac{\mathcal{B}\left(B^{-} \rightarrow\left[K^{+} \pi^{-}\right]_{D} K^{-}\right)+\mathcal{B}\left(B^{+} \rightarrow\left[K^{-} \pi^{+}\right]_{D} K^{+}\right)}{\mathcal{B}\left(B^{-} \rightarrow\left[K^{-} \pi^{+}\right]_{D} K^{-}\right)+\mathcal{B}\left(B^{+} \rightarrow\left[K^{+} \pi^{-}\right]_{D} K^{+}\right)}, \\
A_{\mathrm{ADS}} & \equiv \frac{\mathcal{B}\left(B^{-} \rightarrow\left[K^{+} \pi^{-}\right]_{D} K^{-}\right)-\mathcal{B}\left(B^{+} \rightarrow\left[K^{-} \pi^{+}\right]_{D} K^{+}\right)}{\mathcal{B}\left(B^{-} \rightarrow\left[K^{+} \pi^{-}\right]_{D} K^{-}\right)+\mathcal{B}\left(B^{+} \rightarrow\left[K^{-} \pi^{+}\right]_{D} K^{+}\right)} .
\end{aligned}
$$

The current measurements of these observables are summarized by HFAG ${ }^{7}$. In the analysis of data, usually the GLW and ADS methods are combined and a $\chi^{2}$-fit is done to determine the profile of the phase $\gamma$.

A variant of the $B \rightarrow D K$ method of measuring $\gamma$ is to use the decays $B^{ \pm} \rightarrow D K^{ \pm}$followed by multi-body decays of the $D$-meson, such as $D^{0} \rightarrow K_{S} \pi^{-} \pi^{+}, D^{0} \rightarrow K_{S} K^{-} K^{+}$and $D^{0} \rightarrow K_{S} \pi^{-} \pi^{+} \pi^{0}$, in which a binned Dalitz plot analysis of the decays $D^{0} / \overline{D^{0}} \rightarrow K_{S} \pi^{-} \pi^{+}$was proposed 160,163 . Assuming no $\mathrm{CP}$ asymmetry in $D^{0}$ decays, the amplitude of the $B^{+} \rightarrow D^{0} K^{+} \rightarrow\left(K_{S} \pi^{+} \pi^{-}\right) K^{+}$can be written as

$$
M_{+}=f\left(m_{+}^{2}, m_{-}^{2}\right)+r_{D K} \mathrm{e}^{i\left(\gamma+\delta_{D K}\right)} f\left(m_{-}^{2}, m_{+}^{2}\right),
$$

where $m_{+}^{2}$ and $m_{-}^{2}$ are the squared invariant masses of the $K_{S} \pi^{+}$and $K_{S} \pi^{-}$ combinations in the $D^{0}$ decay, and $f$ is the complex amplitude of the decay $D^{0} \rightarrow K_{S} \pi^{+} \pi^{-}$. The quantities $r_{D K}$ and $\delta_{D K}$ are the relative magnitudes and strong phases of the two amplitudes, already discussed earlier. The amplitude for the charge conjugate $B^{-}$decay is

$$
M_{-}=f\left(m_{-}^{2}, m_{+}^{2}\right)+r_{D K} \mathrm{e}^{i\left(-\gamma+\delta_{D K}\right)} f\left(m_{+}^{2}, m_{-}^{2}\right) .
$$

Once the functional form of $f$ is fixed by a choice of a model for $D^{0} \rightarrow$ $K_{S} \pi^{+} \pi^{-}$decay, the Dalitz distribution for $B^{+}$and $B^{-}$decays can be fitted simultaneously by the expressions for $M_{+}$and $M_{-}$, with $r_{D K}, \delta_{D K}$ and $\gamma\left(\right.$ or $\left.\phi_{3}\right)$ as free parameters. The model-dependence could be removed by a binned Dalitz distribution ${ }^{163}$. This is usually called the GGSZ method, and has been used to determine $\gamma$. The combined fit of both of these methods by CKMfitter 67 yields $\gamma=\left(76.8_{-31.5}^{+30.4}\right)^{\circ}$, to be compared with their overall fit from the CKM unitarity $\gamma=\left(67.6_{-4.5}^{+2.8}\right)^{\circ}$. The corresponding fit by the UTfit group 66 yields $\gamma=(67 \pm 7)^{\circ}$. Thus, we see that within the current experimental error of the direct measurements, which is quite large, also the phase $\gamma$ is compatible with its indirect estimates in the SM. The experimental precision will greatly improve at the LHC, in particular, by using 2-body $B_{s}$-decays. 


\section{4. $B \rightarrow K \pi: S M$ vs. Experiments}

We now discuss the decays $B \rightarrow K \pi$. First, we note that the direct CPasymmetry in the $B \rightarrow K \pi$ decays has now been measured by the BABAR, BELLE and CDF collaborations:

$$
A_{\mathrm{CP}}\left(\pi^{+} K^{-}\right)=\left\{\begin{array}{l}
\left(-10.7 \pm 1.8_{-0.4}^{+0.7}\right) \%[\mathrm{BABAR}], \\
(-9.3 \pm 1.8 \pm 0.8) \%[\mathrm{BELLE}], \\
(-8.6 \pm 2.3 \pm 0.9) \%[\mathrm{CDF}],
\end{array}\right.
$$

to be compared with the predictions of the two factorization-based approaches: $A_{\mathrm{CP}}\left(\pi^{+} K^{-}\right)=(-12.9 \div-21.9) \%[\mathrm{pQCD}] 123$ and $A_{\mathrm{CP}}\left(\pi^{+} K^{-}\right)=$ $(-5.4 \div+13.6) \%[\mathrm{QCD}-\mathrm{F}]{ }^{120}$, with the latter falling short of a satisfactory description of data.

The charged and neutral $B \rightarrow \pi K$ decays have received a lot of theoretical attention. In particular, many ratios involving these decays have been proposed to test the SM 165, 166, 167, 168 and extract useful bounds on the angle $\gamma$, starting from the Fleischer-Mannel bound ${ }^{165}$ :

$$
\sin ^{2} \gamma \leq R \equiv \frac{\tau_{B^{+}}}{\tau_{B_{d}^{0}}} \frac{\mathcal{B}\left(B_{d}^{0} \rightarrow \pi^{-} K^{+}\right)+\mathcal{B}\left(\bar{B}_{d}^{0} \rightarrow \pi^{+} K^{-}\right)}{\mathcal{B}\left(B^{+} \rightarrow \pi^{+} K^{0}\right)+\mathcal{B}\left(B^{-} \rightarrow \pi^{-} \bar{K}^{0}\right)} .
$$

The current experimental average $R=0.899 \pm 0.049$ allows to put a bound: $\gamma<92^{\circ}$ (at $95 \%$ C.L.). This is in agreement with the determination of $\gamma$ from the $B \rightarrow \pi \pi$ and $B \rightarrow D^{(*)} K^{(*)}$ decays given earlier and the indirect unitarity constraints. Thus, both $R$ and $A_{\mathrm{CP}}\left(\pi^{+} K^{-}\right)$are in agreement with the SM. The same is the situation with the Lipkin sum rule ${ }^{167}$ :

$$
R_{L} \equiv 2 \frac{\Gamma\left(B^{+} \rightarrow K^{+} \pi^{0}\right)+\Gamma\left(B^{0} \rightarrow K^{0} \pi^{0}\right)}{\Gamma\left(B^{+} \rightarrow K^{0} \pi^{+}\right)+\Gamma\left(B^{0} \rightarrow K^{+} \pi^{-}\right)}=1+\mathcal{O}\left(\frac{P_{\mathrm{EW}}+T}{P}\right)^{2},
$$

implying significant electroweak penguin contribution in case $R_{L}$ deviates significantly from unit. With the current experimental average $R_{L}=1.071 \pm$ 0.049 , this is obviously not the case. This leaves then the two other ratios $R_{c}$ and $R_{n}$ involving the $B \rightarrow \pi K$ decays of $B^{ \pm}$and $B^{0}$ mesons:

$$
R_{c} \equiv 2 \frac{\mathcal{B}\left(B^{ \pm} \rightarrow \pi^{0} K^{ \pm}\right)}{\mathcal{B}\left(B^{ \pm} \rightarrow \pi^{ \pm} K^{0}\right)}, \quad R_{n} \equiv \frac{1}{2} \frac{\mathcal{B}\left(B_{d}^{0} \rightarrow \pi^{\mp} K^{ \pm}\right)}{\mathcal{B}\left(B_{d}^{0} \rightarrow \pi^{0} K^{0}\right)} .
$$

Their experimental values $R_{c}=1.117 \pm 0.071$ and $R_{n}=0.980 \pm 0.067$ are to be compared with the current SM-based estimates ${ }^{164} R_{c}=1.14 \pm 0.05$ and $R_{n}=1.11_{-0.05}^{+0.04}$. This implies $R_{c}(\mathrm{SM})-R_{c}(\operatorname{Exp})=0.02 \pm 0.09$ and $R_{n}(\mathrm{SM})-R_{n}(\mathrm{Exp})=0.13 \pm 0.08$. Possible deviations from the SM, if confirmed, would imply new physics, advocated in this context, in particular, 
by Yoshikawa ${ }^{169}$, Beneke and Neubert 170 and Buras et al. ${ }^{164}$. However, as of now, one has to conclude that SM is in agreement with the measurements of both $R_{c}$ and $R_{n}$.

Finally, a bound on $\mathcal{B}\left(B^{0} \rightarrow K^{0} \overline{K^{0}}\right)$ based on $S U(3)$ and $B \rightarrow \pi \pi$ data, obtained recently by Fleischer and Recksiegel ${ }^{171}$, yielding $\mathcal{B}\left(B^{0} \rightarrow\right.$ $\left.K^{0} \bar{K}^{0}\right)<1.5 \times 10^{-6}$ is well satisfied by the current world average ${ }^{7} \mathcal{B}\left(B^{0} \rightarrow\right.$ $\left.K^{0} \bar{K}^{0}\right)=\left(0.96_{-0.19}^{+0.21}\right) \times 10^{-6}$.

\section{6. $B_{s}^{0}$ Physics: Eldorado for the Tevatron and the LHC}

The main goal of $b$ physics at the hadron colliders Tevatron and the LHC is to chart out the physics of the $B_{s}^{0}$ and $B_{c}^{ \pm}$mesons and of the $b$-baryons. The current information on the spectroscopic and decay characteristics of these hadrons is still very much in the offing, though clearly the two Tevatron experiments have made some incisive inroads in these otherwise uncharted territories. Despite the overwhelming performance of the $B$ factory experiments, there still remain a few landmark measurements to be carried out involving $B_{d}^{0}$ and $B^{ \pm}$mesons. These include, precise measurements of the $\mathrm{CP}$ asymmetries in the penguin-dominated exclusive decays, quantitative determinations of the Wilson coefficients in the effective theory for weak decays $\left(C_{7}, C_{8}, C_{9}, C_{10}\right)$, which will be made possible by the precise measurements of the radiative and semileptonic decays $B \rightarrow\left(X_{s}, K^{*}\right) \gamma$ and $B \rightarrow\left(X_{s}, K, K^{*}\right) \ell^{+} \ell^{-}$. It is challenging to measure the inclusive decays at the LHC, but certainly exclusive decays will be well measured.

In this section, a brief list of some selected $b$ physics topics to be studied at the LHC is given and discussed.

- $B_{s}^{0}-\overline{B_{s}^{0}}$ Mixing.

Apart from the precise measurement of $\Delta M_{B_{s}}=(17.77 \pm 0.10 \pm 0.07)$ $(\mathrm{ps})^{-1}$ by the CDF collaboration, there are two other quantities still to be measured in this complex: Lifetime difference $\Delta \Gamma_{B_{s}}$ and the phase $\phi_{s}$. These quantities have been calculated to a high precision in the SM ${ }^{172}$. A recent update of this work yields 71

$$
\begin{aligned}
\frac{\Delta \Gamma_{B_{s}}}{\Delta M_{B_{s}}} & =(49.7 \pm 9.4) \times 10^{-4} ; \quad \Delta \Gamma_{B_{s}}=(0.096 \pm 0.039)(\mathrm{ps})^{-1}, \\
\phi_{s} & =(4.2 \pm 1.4) \times 10^{-3}=0.24^{\circ} \pm 0.08^{\circ} .
\end{aligned}
$$

The current measurements of these quantities from the D0 collaboration are 173

$$
\Delta \Gamma_{B_{s}}=\left(0.12_{-0.10}^{+0.08} \pm 0.02\right)(\mathrm{ps})^{-1}\left(\text { assuming } \phi_{s}=0\right),
$$


and

$$
\Delta \Gamma_{B_{s}}=(0.17 \pm 0.09 \pm 0.02)(\mathrm{ps})^{-1} ; \phi_{s}=-0.79 \pm 0.56_{-0.01}^{+0.14} .
$$

The corresponding measurement (assuming $\phi_{s}=0$ ) for $\Delta \Gamma_{B_{s}}$ from CDF is $174 \Delta \Gamma_{B_{s}}=\left(0.076_{-0.063}^{+0.059} \pm 0.006\right)(\mathrm{ps})^{-1}$, where the first error is statistical and the second systematic. At the $\mathrm{LHCb}^{12}$, one anticipates a statistical sensitivity of $\sigma\left(\sin \phi_{s}\right) \sim 0.031$ and $\sigma\left(\Delta \Gamma_{s} / \Gamma_{s}\right) \sim 0.011$, assuming an integrated luminosity of $2(\mathrm{fb})^{-1}$ and using the decay $B_{s} \rightarrow J / \psi \phi$. This sensitivity will be improved by accumulating more data and adding the $\mathrm{CP}$ modes $B_{s} \rightarrow J / \psi \phi$ and the pure CP modes $B_{s} \rightarrow J / \psi \eta$ and $B_{s} \rightarrow J / \psi \eta^{\prime}$. The ATLAS and CMS sensitivities on $\phi_{s}$ are expected to be somewhat worse by typically a factor 2. Thus, experiments at the LHC will be able to test the SM estimates for both the quantities $\Delta \Gamma_{B_{s}}$ and $\phi_{s}$.

- Precise measurement of the phase $\gamma$.

At the LHC one can combine the methods of determining the phase $\gamma$ already in use at the $B$ factories involving the decays $B^{+} \rightarrow D^{(*)} K^{+}$and $B^{0} \rightarrow D K^{(*)}$, with the decay $B_{s}^{0} \rightarrow D_{s} K$. In addition, one can use the U-spin symmetry arguments advocated, in particular, by Fleischer ${ }^{175}$, to combine data from the decays $B_{d}^{0} \rightarrow \pi^{+} \pi^{-}$and $B_{s}^{0} \rightarrow K^{+} K^{-}$to constrain $\gamma$. An educated guess 176 is that a sensitivity $\sigma(\gamma) \simeq 4^{\circ}$ with $2(\mathrm{fb})^{-1}$ of data is reachable at the $\mathrm{LHCb}$, improving to $\sigma(\gamma) \simeq 2.4^{\circ}$ with $10(\mathrm{fb})^{-1}$. This will result in an order of magnitude improvement over the current precision on this phase. Modest improvements are also anticipated for the other two phases $\alpha$ and $\beta$ at the LHC.

- Leptonic decay $B_{s}^{0} \rightarrow \mu^{+} \mu^{-}$

New and improved upper limits have been presented by the CDF 177 and D0 178 collaborations for the decays $B_{s}^{0} \rightarrow \mu^{+} \mu^{-}$and $B_{d}^{0} \rightarrow \mu^{+} \mu^{-}$. They are as follows (at 95\% C.L.)

$$
\begin{aligned}
& \mathcal{B}\left(B_{s}^{0} \rightarrow \mu^{+} \mu^{-}\right)<9.3[5.8] \times 10^{-8} \quad \mathrm{D} 0[\mathrm{CDF}], \\
& \mathcal{B}\left(B_{d}^{0} \rightarrow \mu^{+} \mu^{-}\right)<1.8 \times 10^{-8} \quad[\mathrm{CDF}] .
\end{aligned}
$$

The CDF and DO upper limits have been combined to yield $5 \mathcal{B}\left(B_{s}^{0} \rightarrow\right.$ $\left.\mu^{+} \mu^{-}\right)<4.5 \times 10^{-8}$, to be compared with the SM predictions 179 $\mathcal{B}\left(B_{s}^{0} \rightarrow \mu^{+} \mu^{-}\right)=3.4 \times 10^{-9}$ and $\mathcal{B}\left(B_{d}^{0} \rightarrow \mu^{+} \mu^{-}\right)=1.0 \times 10^{-10}$ with $\pm 15 \%$ theoretical uncertainty. Hence, currently there is no sensitivity for the SM decay rate. However, as the leptonic branching ratios probe the Higgs sector in beyond-the-SM scenarios, such as supersymmetry, and they 
depend sensitively on $\tan \beta$, the Tevatron upper limit on $\mathcal{B}\left(B_{s}^{0} \rightarrow \mu^{+} \mu^{-}\right)$ probes the large $\tan \beta$ parameter space, though the precise constraints are model dependent 180,181 . At the LHC, the two main collider experiments ATLAS and CMS will reach the SM sensitivity, certainly with the higher LHC luminosity, $L_{\mathrm{LHC}}=10^{34} \mathrm{~cm}^{-2} \mathrm{~s}^{-1}$, as the decay $B_{s}^{0} \rightarrow \mu^{+} \mu^{-}$remains triggerable with the high luminosity.

- Charmless non-leptonic $B_{s} \rightarrow h_{1} h_{2}$ decays.

The experimental program to study non-leptonic decays $B_{s}^{0} \rightarrow h_{1} h_{2}$ has started (here $h_{1,2}$ stand for charmless light vector or pseudoscalar mesons) with first measurements for the branching ratios $B_{s}^{0} \rightarrow K^{+} \pi^{-}$and $B_{s}^{0} \rightarrow K^{+} K^{-}$made available recently by the CDF collaboration 9,10 . Remarkably, the first direct $\mathrm{CP}$ asymmetry involving the decay $B_{s}^{0} \rightarrow K^{+} \pi^{-}$ and its $\mathrm{CP}$ conjugate mode reported by $\mathrm{CDF}$ is found to be large, with $A_{\mathrm{CP}}\left(\overline{B_{s}^{0}} \rightarrow K^{+} \pi^{-}\right)=(39 \pm 15 \pm 8) \%$. This large CP asymmetry was predicted by Lipkin 182 based on SU(3) symmetry arguments. This already tests various dynamical models, such as QCDF ${ }^{183}$, SCET ${ }^{184}$ and pQCD ${ }^{185}$. With the ongoing $b$-physics program at the Tevatron, but, in particular, with the onset of the LHC, we expect a wealth of data involving the decays of the hitherto less studied $B_{s}^{0}$ meson. The charmless $B_{s}^{0} \rightarrow h_{1} h_{2}$ decays are also important for the $\mathrm{CP}$ asymmetry studies and the determination of the inner angles of the unitarity triangle. As already stated, a number of charmless decays $B_{s}^{0} \rightarrow h_{1} h_{2}$ can be related to the $B_{d}^{0} \rightarrow h_{1} h_{2}$ decays using $\mathrm{SU}(3)$ (or U-spin) symmetry, and hence data on these decays can be combined to test the underlying standard model and search for physics beyond the SM under less (dynamical) model-dependent conditions. Anticipating the experimental developments, many studies have been devoted to the interesting charmless $B_{s}^{0} \rightarrow h_{1} h_{2}$ decays, waiting to be tested at the LHC.

\section{Summary and Outlook}

Summarizing, dedicated experiments carried out over several decades combined with progress in theoretical techniques embedded in QCD have enabled a precise determination of the CKM matrix elements. The knowledge of the third row and the third column of $V_{\mathrm{CKM}}$ has come from $b$-physics, which we discussed at length in this review. Of these, precise determination of $V_{c b}$ and $V_{u b}$ required good control over the perturbative and nonperturbative aspects of QCD. The current precision on the direct determination of $V_{t b}$ from the decay $t \rightarrow b W$ is limited by statistics and this 
will vastly improve from the top quark studies at the LHC and later at the ILC. The determination of $V_{t s}$ and $V_{t d}$ require not only precise knowledge of QCD in $b$ decays but implicitly also the assumption of the CKM unitarity, as they are determined from the loop-induced $b \rightarrow s$ and $b \rightarrow d$ transitions. Their current best measurement is through the mass differences $\Delta M_{B_{s}}$ and $\Delta M_{B_{d}}$, and the precision on these matrix elements (typically $10 \%$ ) is completely dominated by theory. A complementary determination of $V_{t d}$ and $V_{t s}$ is also at hand from the radiative penguin transitions $b \rightarrow(d, s) \gamma$ and the exclusive decays $B \rightarrow\left(K^{*}, \rho, \omega\right) \gamma$, but the current precision is limited by both experimental statistics and non-perturbative aspects of QCD, such as the transition form factors in exclusive decays. This surely will improve over the next several years.

Experiments have also firmly established the phenomenon of $\mathrm{CP}$ violation in the $K$ and $B$ meson sectors. The various $\mathrm{CP}$ asymmetries in these decays are found compatible with each other and, with some help from $\mathrm{QCD}$, have a consistent interpretation in terms of the single complex phase of the CKM matrix. Again, in principle, there is ample room also for beyond-the-SM weak phases, which would lead to very different patterns of $\mathrm{CP}$ asymmetries in the tree-dominated versus loop-dominated transitions. This has not been borne out by experiments at the $B$ factories. While the current data is not equivocal on all the decay channels, and the dynamical aspects of not all the measured $B$-meson decays are quantitatively understood, the experimental case for the extra weak-phases is rather weak. Ongoing experiments at the $B$ factories are expected to significantly reduce the errors on the quantities $S_{f}$ and $C_{f}$ in penguin-dominated decays to settle the issue of new weak phases in $B$ decays.

From the foregoing one has to tentatively conclude that the CKM paradigm is now firmly established as the dominant mechanism of flavor transitions in the quark sector. Whether future experiments, such as at the LHC and (Super) $B$ factories, will force us to modify this paradigm remains to be seen. We expect on theoretical grounds that there is New Physics, probably just around the corner, to solve the outstanding issue of the gauge hierarchy. The resolution of this problem together with the unification of the gauge couplings and the search of viable candidate(s) for dark matter requires a TeV scale New Physics. Assuming that supersymmetry is the most viable candidate for the impending New Physics to be discovered by experiments at the LHC, the central issue in the LHC era would be to pin down the underlying flavor aspects of this theory. However, if the current experimental trend is any indicator, then very likely the New Physics will 
be of the minimal flavor violating type, or something akin to it. Verifying this and, more importantly, quantifying the subdominant flavor structures in supersymmetry (or its alternative) is the next task to which theory and experiment have to gear themselves up.

\section{Acknowledgments}

I would like to congratulate Professor Riazuddin and the faculty of the National Centre for Physics in Islamabad on the auspicious occasion of the inauguration of the new campus. I have thoroughly enjoyed this symposium marking the inaugural and thank Professor Riazuddin and his staff for their kind hospitality. Thanks are also due to Alexander Parkhomenko for critically reading this manuscript and his helpful remarks.

\section{References}

1. N. Cabibbo, Phys. Rev. Lett. 10 (1963) 531

2. M. Kobayashi and T. Maskawa, Prog. Theor. Phys. 49 (1973) 652.

3. E. Barberio, in Proc. of the XXIII International Symposium on Lepton and Photon Interactions at High Energy, Aug. 13-18, 2007, Daego, Korea. Below this is termed as the LP-2007 Symposium (http://chep.knu.ac.kr/LP07/).

4. D. Brown, in Proc. of the LP-2007 Symposium.

5. M. Nakao, in Proc. of the LP-2007 Symposium.

6. J. Wicht [BELLE Collaboration], arXiv:0711.0271 [hep-ex].

7. Heavy Flavor Averaging Group [HFAG]. The most recent HFAG averages can be seen in the eprint: E. Barberio et al. [arXiv:0704.3575 (hep-ex)] and from the HFAG website (http://www.slac.stanford.edu/xorg/hfag/).

8. V. M. Abazov et al. [D0 Collaboration], Phys. Rev. Lett. 97, 021802 (2006) [arXiv:hep-ex/0603029].

9. A. Abulencia et al. [CDF - Run II Collaboration], Phys. Rev. Lett. 97, 062003 (2006) [arXiv:hep-ex/0606027].

10. M. Morello [CDF Collaboration], Nucl. Phys. Proc. Suppl. 170, 39 (2007) [arXiv:hep-ex/0612018].

11. A. Abulencia et al. [CDF Collaboration], Phys. Rev. Lett. 97, 211802 (2006) [arXiv:hep-ex/0607021].

12. For the LHCb detector details and the physics potential, see the LHCb website: http://lhcb.cern.ch/lhcb/.

13. W. M. Yao et al. [Particle Data Group], J. Phys. G 33, 1 (2006).

14. See, for example, the proceedings of the Workshop on Theory, Phenomenology and Experiments in Heavy Flavor Physics, Capri, Italy, 29-31 May 2006; Nucl. Phys. B, Proc. Suppl. 159 (2006) 213 p (Editors: G. Ricciardi and C. Sciacca).

15. L. Wolfenstein, Phys. Rev. Lett. 51, 1945 (1983).

16. A. J. Buras, M. E. Lautenbacher and G. Ostermaier, Phys. Rev. D 50, 3433 (1994) [hep-ph/9403384].

17. J. C. Hardy and I. S. Towner, Phys. Rev. Lett. 94, 092502 (2005) [arXiv:nuclth/0412050].

18. G. Savard et al., Phys. Rev. Lett. 95, 102501 (2005). 
19. H. Leutwyler and M. Roos, Z. Phys. C 25, 91 (1984).

20. For a recent review of Lattice QCD predictions for D-decays, see A. S. Kronfeld [Fermilab Lattice Collaboration], J. Phys. Conf. Ser. 46, 147 (2006) [arXiv:hep-lat/0607011].

21. See, for example, A. V. Manohar and M. B. Wise, Cambridge Monogr. Part. Phys. Nucl. Phys. Cosmol. 10 (2000) 1.

22. J. Chay, H. Georgi, and B. Grinstein, Phys. Lett. B247 (1990) 399; M. Voloshin and M. Shifman, Sov. J. Nucl. Phys. 41 (1985) 120.

23. I.I. Bigi et al., Phys. Lett. B293 (1992) 430; Phys. Lett B297 (1993) 477 (E); Phys. Rev. Lett. 71 (1993) 496.

24. A.V. Manohar and M.B. Wise, Phys. Rev. D49 (1994) 1310.

25. B. Blok, L. Koyrakh, M. A. Shifman and A. I. Vainshtein, Phys. Rev. D 49, 3356 (1994); Phys. Rev. D 50, 3572 (1994) (E) [hep-ph/9307247].

26. T. Mannel, Nucl. Phys. B 413, 396 (1994) [hep-ph/9308262].

27. C. W. Bauer, Z. Ligeti, M. Luke, A. V. Manohar and M. Trott, Phys. Rev. D 70, 094017 (2004) [hep-ph/0408002].

28. P. Gambino and N. Uraltsev, Eur. Phys. J. C 34, 181 (2004) [hep$\mathrm{ph} / 0401063]$.

29. N. Isgur and M. B. Wise, Phys. Lett. B 232, 113 (1989). Phys. Lett. B 237, 527 (1990).

30. M. E. Luke, Phys. Lett. B 252, 447 (1990).

31. J. D. Bjorken, SLAC-PUB-5278 Talk given at Les Rencontre de la Valle d'Aoste, La Thuile, Italy, Mar 18-24, 1990.

32. N. Uraltsev, Phys. Lett. B 501, 86 (2001) [hep-ph/0011124]; J. Phys. G 27, 1081 (2001) [hep-ph/0012336].

33. F. Jugeau, A. Le Yaouanc, L. Oliver and J. C. Raynal, Phys. Rev. D 71, 054031 (2005) [arXiv:hep-ph/0407176].

34. J. P. Archambault and A. Czarnecki, Phys. Rev. D 70, 074016 (2004) [hep$\mathrm{ph} / 0408021]$.

35. S. Hashimoto, A. S. Kronfeld, P. B. Mackenzie, S. M. Ryan and J. N. Simone, Phys. Rev. D 66, 014503 (2002) [hep-ph/0110253].

36. M. Luke, eConf C0304052, WG107 (2003) [hep-ph/0307378].

37. M. Neubert, Phys. Rev. D49 (1994) 3392; D49 (1994) 4623; T. Mannel and M. Neubert, Phys. Rev. D50 (1994) 2037; I.I. Bigi et al., Int. J. Mod. Phys. A9 (1994) 2467.

38. A. K. Leibovich, I. Low and I. Z. Rothstein, Phys. Rev. D 61, 053006 (2000) [hep-ph/9909404]; Phys. Lett. B 486, 86 (2000) [hep-ph/0005124]; Phys. Lett. B 513, 83 (2001) [hep-ph/0105066].

39. M. Neubert, Phys. Lett. B 513, 88 (2001) [hep-ph/0104280].

40. C. W. Bauer, M. E. Luke and T. Mannel, Phys. Rev. D 68, 094001 (2003) [hep-ph/0102089].

41. G. P. Korchemsky and G. Sterman, Phys. Lett. B 340, 96 (1994) [hepph/9407344].

42. C. W. Bauer, Z. Ligeti and M. E. Luke, Phys. Rev. D 64, 113004 (2001) [arXiv:hep-ph/0107074].

43. C. W. Bauer, S. Fleming and M. E. Luke, Phys. Rev. D 63, 014006 (2001) 
[arXiv:hep-ph/0005275].

44. C. W. Bauer, S. Fleming, D. Pirjol and I. W. Stewart, Phys. Rev. D 63, 114020 (2001) [arXiv:hep-ph/0011336].

45. C. W. Bauer, D. Pirjol and I. W. Stewart, Phys. Rev. D 65, 054022 (2002) [arXiv:hep-ph/0109045].

46. M. Beneke, A. P. Chapovsky, M. Diehl and T. Feldmann, Nucl. Phys. B 643, 431 (2002) [arXiv:hep-ph/0206152].

47. B. O. Lange, M. Neubert and G. Paz, Phys. Rev. D 72, 073006 (2005) [arXiv:hep-ph/0504071].

48. J. R. Andersen and E. Gardi, JHEP 0601, 097 (2006) [arXiv:hep$\mathrm{ph} / 0509360]$.

49. P. Ball and R. Zwicky, Phys. Rev. D 71, 014015 (2005) [arXiv:hep$\mathrm{ph} / 0406232]$.

50. E. Dalgic, A. Gray, M. Wingate, C. T. H. Davies, G. P. Lepage and J. Shigemitsu, Phys. Rev. D 73, 074502 (2006) [Erratum-ibid. D 75, 119906 (2007)] [arXiv:hep-lat/0601021].

51. M. Okamoto et al., Nucl. Phys. Proc. Suppl. 140, 461 (2005) [arXiv:heplat/0409116].

52. A. Abada, D. Becirevic, P. Boucaud, J. P. Leroy, V. Lubicz and F. Mescia, Nucl. Phys. B 619, 565 (2001) [arXiv:hep-lat/0011065].

53. B. Grinstein and D. Pirjol, Phys. Rev. D 70, 114005 (2004) [arXiv:hep$\mathrm{ph} / 0404250]$.

54. D. E. Acosta et al. [CDF Collaboration], Phys. Rev. Lett. 95, 102002 (2005) [arXiv:hep-ex/0505091].

55. J. A. Aguilar-Saavedra et al. [ECFA/DESY LC Physics Working Group], arXiv:hep-ph/0106315.

56. A. J. Buras, W. Slominski and H. Steger, Nucl. Phys. B 245, 369 (1984).

57. A. J. Buras, M. Jamin and P. H. Weisz, Nucl. Phys. B 347, 491 (1990).

58. T. Inami and C. S. Lim, Prog. Theor. Phys. 65, 297 (1981) [Erratum-ibid. 65, $1772(1981)]$

59. M. Jamin and B. O. Lange, Phys. Rev. D 65, 056005 (2002) [hep$\mathrm{ph} / 0108135]$.

60. A. A. Penin and M. Steinhauser, Phys. Rev. D 65, 054006 (2002) [hep$\mathrm{ph} / 0108110]$.

61. J. G. Korner, A. I. Onishchenko, A. A. Petrov and A. A. Pivovarov, Phys. Rev. Lett. 91, 192002 (2003) [arXiv:hep-ph/0306032].

62. S. Aoki et al. [JLQCD Collaboration], Phys. Rev. Lett. 91, 212001 (2003) [arXiv:hep-ph/0307039].

63. A. Gray et al. [HPQCD Collaboration], Phys. Rev. Lett. 95, 212001 (2005) [arXiv:hep-lat/0507015].

64. M. Okamoto, PoS LAT2005, 013 (2006) [arXiv:hep-lat/0510113].

65. E. Dalgic et al., Phys. Rev. D 76, 011501 (2007) [arXiv:hep-lat/0610104].

66. M. Bona et al. [UTfit Collaboration], arXiv:0707.0636 [hep-ph].

67. J. Charles et al. [CKMfitter Group], Eur. Phys. J. C 41, 1 (2005) [arXiv:hepph/0406184]. Updates are taken from the CKMFitter Working Group URL: http://ckmfitter.in2p3.fr. 
68. M. Ciuchini and L. Silvestrini, Phys. Rev. Lett. 97, 021803 (2006) [arXiv:hep$\mathrm{ph} / 0603114$.

69. M. Endo and S. Mishima, Phys. Lett. B 640, 205 (2006) [arXiv:hep$\mathrm{ph} / 0603251]$.

70. Z. Ligeti, M. Papucci and G. Perez, Phys. Rev. Lett. 97, 101801 (2006) [arXiv:hep-ph/0604112].

71. A. Lenz and U. Nierste, JHEP 0706, 072 (2007) [arXiv:hep-ph/0612167].

72. A. Ali and D. London, Eur. Phys. J. C 18, 665 (2001) [arXiv:hep$\mathrm{ph} / 0012155]$.

73. A. Ali and D. London, Eur. Phys. J. C 9, 687 (1999) [arXiv:hep-ph/9903535]; A. J. Buras, P. Gambino, M. Gorbahn, S. Jager and L. Silvestrini, Phys. Lett. B 500, 161 (2001) [arXiv:hep-ph/0007085]; G. D’Ambrosio, G. F. Giudice, G. Isidori and A. Strumia, Nucl. Phys. B 645, 155 (2002) [arXiv:hep$\mathrm{ph} / 0207036]$.

74. A. Ali and Z. Z. Aydin, Nucl. Phys. B 148, 165 (1979).

75. G. Buchalla, A. J. Buras and M. E. Lautenbacher, Rev. Mod. Phys. 68, 1125 (1996) [arXiv:hep-ph/9512380].

76. T. Hurth, Rev. Mod. Phys. 75, 1159 (2003) [arXiv:hep-ph/0212304].

77. C. Bobeth, M. Misiak and J. Urban, Nucl. Phys. B 574, 291 (2000) [hepph/9910220].

78. M. Misiak and M. Steinhauser, Nucl. Phys. B 683, 277 (2004) [hep$\mathrm{ph} / 0401041]$.

79. K. G. Chetyrkin, M. Misiak and M. Münz, Phys. Lett. B 400, 206 (1997) [Erratum-ibid. B 425, 414 (1998)] [hep-ph/9612313].

80. M. Gorbahn and U. Haisch, Nucl. Phys. B 713, 291 (2005) [arXiv:hepph/0411071].

81. M. Gorbahn, U. Haisch and M. Misiak, Phys. Rev. Lett. 95, 102004 (2005) [arXiv:hep-ph/0504194].

82. M. Czakon, U. Haisch and M. Misiak, JHEP 0703, 008 (2007) [arXiv:hep$\mathrm{ph} / 0612329]$.

83. C. Greub, T. Hurth and D. Wyler, Phys. Rev. D 54, 3350 (1996) [hepph/9603404].

84. I. Blokland, A. Czarnecki, M. Misiak, M. Slusarczyk and F. Tkachov, Phys. Rev. D 72, 033014 (2005) [arXiv:hep-ph/0506055].

85. H. M. Asatrian, A. Hovhannisyan, V. Poghosyan, T. Ewerth, C. Greub and T. Hurth, Nucl. Phys. B 749, 325 (2006) [arXiv:hep-ph/0605009].

86. H.M. Asatrian, T. Ewerth, A. Ferroglia, P. Gambino, C. Greub and G. Ossola, in preparation.

87. A. J. Buras, A. Czarnecki, M. Misiak and J. Urban, Nucl. Phys. B 611, 488 (2001) [arXiv:hep-ph/0105160].

88. A. J. Buras, A. Czarnecki, M. Misiak and J. Urban, Nucl. Phys. B 631, 219 (2002) [arXiv:hep-ph/0203135].

89. K. Bieri, C. Greub and M. Steinhauser, Phys. Rev. D 67, 114019 (2003) [arXiv:hep-ph/0302051].

90. M. Misiak and M. Steinhauser, Nucl. Phys. B 764, 62 (2007) [arXiv:hep$\mathrm{ph} / 0609241]$. 
91. M. Misiak et al., Phys. Rev. Lett. 98, 022002 (2007) [arXiv:hep-ph/0609232].

92. A. Ali and C. Greub, Z. Phys. C 49, 431 (1991); Phys. Lett. B 361, 146 (1995) [hep-ph/9506374]; N. Pott, Phys. Rev. D 54, 938 (1996) [hep-ph/9512252].

93. K. Melnikov and A. Mitov, Phys. Lett. B 620, 69 (2005) [arXiv:hep$\mathrm{ph} / 0505097$.

94. J. R. Ellis, S. Heinemeyer, K. A. Olive, A. M. Weber and G. Weiglein, JHEP 0708, 083 (2007) [arXiv:0706.0652 [hep-ph]].

95. T. Becher and M. Neubert, Phys. Rev. Lett. 98, 022003 (2007) [arXiv:hepph/0610067].

96. B. Grinstein, M. J. Savage and M. B. Wise, Nucl. Phys. B 319, 271 (1989).

97. F. Kruger and L. M. Sehgal, Phys. Lett. B 380, 199 (1996) [arXiv:hepph/9603237].

98. A. F. Falk, M. E. Luke and M. J. Savage, Phys. Rev. D 49, 3367 (1994) [arXiv:hep-ph/9308288].

99. A. Ali, G. Hiller, L. T. Handoko and T. Morozumi, Phys. Rev. D 55, 4105 (1997) [arXiv:hep-ph/9609449].

100. C. W. Bauer and C. N. Burrell, Phys. Rev. D 62, 114028 (2000) [arXiv:hepph/9911404]; Phys. Lett. B 469, 248 (1999) [arXiv:hep-ph/9907517].

101. J. W. Chen, G. Rupak and M. J. Savage, Phys. Lett. B 410, 285 (1997) [arXiv:hep-ph/9705219]; G. Buchalla, G. Isidori and S. J. Rey, Nucl. Phys. B 511, 594 (1998) [arXiv:hep-ph/9705253].

102. A. Ali, E. Lunghi, C. Greub and G. Hiller, Phys. Rev. D 66, 034002 (2002) [hep-ph/0112300].

103. H. H. Asatryan, H. M. Asatrian, C. Greub and M. Walker, Phys. Rev. D 65, 074004 (2002) [hep-ph/0109140]. H. H. Asatrian, H. M. Asatrian, C. Greub and M. Walker, Phys. Lett. B 507, 162 (2001) [hep-ph/0103087].

104. A. Ghinculov, T. Hurth, G. Isidori and Y. P. Yao, Nucl. Phys. B 685, 351 (2004) [hep-ph/0312128].

105. C. Bobeth, P. Gambino, M. Gorbahn and U. Haisch, JHEP 0404, 071 (2004) [arXiv:hep-ph/0312090].

106. A. Ali and G. Hiller, Phys. Rev. D 60, 034017 (1999) [arXiv:hep$\mathrm{ph} / 9807418]$.

107. K. S. M. Lee, Z. Ligeti, I. W. Stewart and F. J. Tackmann, Phys. Rev. D 74, 011501 (2006) [arXiv:hep-ph/0512191].

108. Z. Ligeti and F. J. Tackmann, Phys. Lett. B 653, 404 (2007) [arXiv:0707.1694 [hep-ph]].

109. A. Ali, G. F. Giudice and T. Mannel, Z. Phys. C 67, 417 (1995) [hep$\mathrm{ph} / 9408213]$.

110. G. Hiller and F. Krüger, Phys. Rev. D 69, 074020 (2004) [hep-ph/0310219].

111. P. Gambino, U. Haisch and M. Misiak, Phys. Rev. Lett. 94, 061803 (2005) [arXiv:hep-ph/0410155].

112. A. Ali, T. Mannel and T. Morozumi, Phys. Lett. B 273, 505 (1991).

113. A. Ghinculov, T. Hurth, G. Isidori and Y. P. Yao, Nucl. Phys. B 648, 254 (2003) [hep-ph/0208088].

114. H. M. Asatrian, K. Bieri, C. Greub and A. Hovhannisyan, Phys. Rev. D 66, 094013 (2002) [hep-ph/0209006]. 
115. G. Burdman, Phys. Rev. D 57, 4254 (1998) [hep-ph/9710550].

116. A. Ali, P. Ball, L. T. Handoko and G. Hiller, Phys. Rev. D 61, 074024 (2000) [hep-ph/9910221].

117. M. Beneke and T. Feldmann, Nucl. Phys. B 592, 3 (2001) [hep-ph/0008255].

118. M. Beneke, T. Feldmann and D. Seidel, Nucl. Phys. B 612 (2001) 25 [hep$\mathrm{ph} / 0106067$.

119. A. Ali, G. Kramer and G. h. Zhu, Eur. Phys. J. C 47, 625 (2006) [arXiv:hep$\mathrm{ph} / 0601034]$.

120. M. Beneke, G. Buchalla, M. Neubert and C. T. Sachrajda, Phys. Rev. Lett. 83, 1914 (1999) [arXiv:hep-ph/9905312].

121. M. Beneke, G. Buchalla, M. Neubert and C. T. Sachrajda, Nucl. Phys. B 606, 245 (2001) [arXiv:hep-ph/0104110].

122. A. Ishikawa et al., Phys. Rev. Lett. 96, 251801 (2006) [arXiv:hepex/0603018].

123. Y. Y. Keum, H. n. Li and A. I. Sanda, Phys. Lett. B 504, 6 (2001) [hepph/0004004]; Y. Y. Keum and A. I. Sanda, Phys. Rev. D 67, 054009 (2003) [hep-ph/0209014].

124. A. Ali and A. Y. Parkhomenko, Eur. Phys. J. C 23, 89 (2002) [arXiv:hep$\mathrm{ph} / 0105302]$.

125. S. W. Bosch and G. Buchalla, Nucl. Phys. B 621, 459 (2002) [arXiv:hep$\mathrm{ph} / 0106081]$.

126. A. L. Kagan and M. Neubert, Phys. Lett. B 539, 227 (2002) [hep$\mathrm{ph} / 0110078]$.

127. A. Ali, E. Lunghi and A. Y. Parkhomenko, Phys. Lett. B 595, 323 (2004) [arXiv:hep-ph/0405075].

128. S. W. Bosch and G. Buchalla, JHEP 0501, 035 (2005) [arXiv:hep$\mathrm{ph} / 0408231]$.

129. M. Beneke, T. Feldmann and D. Seidel, Eur. Phys. J. C 41, 173 (2005) [arXiv:hep-ph/0412400].

130. A. Ali and A. Parkhomenko, arXiv:hep-ph/0610149.

131. P. Ball and R. Zwicky, JHEP 0604, 046 (2006) [arXiv:hep-ph/0603232].

132. Y. Y. Keum, M. Matsumori and A. I. Sanda, Phys. Rev. D 72, 014013 (2005) [arXiv:hep-ph/0406055].

133. C. D. Lu, M. Matsumori, A. I. Sanda and M. Z. Yang, Phys. Rev. D 72 , 094005 (2005) [Erratum-ibid. D 73, 039902 (2006)] [arXiv:hep-ph/0508300].

134. J. g. Chay and C. Kim, Phys. Rev. D 68, 034013 (2003) [arXiv:hep$\mathrm{ph} / 0305033]$.

135. T. Becher, R. J. Hill and M. Neubert, Phys. Rev. D 72, 094017 (2005) [arXiv:hep-ph/0503263].

136. M. Beneke and T. Feldmann, Nucl. Phys. B 685, 249 (2004) [arXiv:hep$\mathrm{ph} / 0311335]$.

137. B. O. Lange and M. Neubert, Nucl. Phys. B 690, 249 (2004) [Erratum-ibid. B 723, 201 (2005)] [arXiv:hep-ph/0311345].

138. M. Beneke, Y. Kiyo and D. s. Yang, Nucl. Phys. B 692, 232 (2004) [arXiv:hep-ph/0402241].

139. M. Beneke and D. Yang, Nucl. Phys. B 736, 34 (2006) [arXiv:hep- 
$\mathrm{ph} / 0508250]$.

140. R. J. Hill, T. Becher, S. J. Lee and M. Neubert, JHEP 0407, 081 (2004) [arXiv:hep-ph/0404217].

141. T. Becher and R. J. Hill, JHEP 0410, 055 (2004) [arXiv:hep-ph/0408344].

142. A. Ali, B. D. Pecjak and C. Greub, arXiv:0709.4422 [hep-ph].

143. A. Ali and V. M. Braun, Phys. Lett. B 359, 223 (1995) [hep-ph/9506248].

144. Y. Li, C. D. Lu and W. Wang, arXiv:0711.0497 [hep-ph].

145. K. Koneke [Babar Collaboration], arXiv:0710.4855 [hep-ex].

146. M. Gronau and D. London, Phys. Rev. Lett. 65, 3381 (1990).

147. A. J. Buras and R. Fleischer, Phys. Lett. B 341, 379 (1995) [hep$\mathrm{ph} / 9409244]$.

148. N. G. Deshpande and X. G. He, Phys. Rev. Lett. 74 (1995) 26 [Erratum-ibid. 74 (1995) 4099] [hep-ph/9408404]. M. Gronau, O. F. Hernandez, D. London and J. L. Rosner, Phys. Rev. D 52, 6374 (1995) [hep-ph/9504327].

149. M. Gronau and J. L. Rosner, Phys. Rev. D 66, 053003 (2002) [Erratum-ibid. D 66, 119901 (2002)] [hep-ph/0205323].

150. M. Ciuchini, E. Franco, G. Martinelli, M. Pierini and L. Silvestrini, Phys. Lett. B 515, 33 (2001) [hep-ph/0104126].

151. A. J. Buras and L. Silvestrini, Nucl. Phys. B 569, 3 (2000) [hep-ph/9812392].

152. A. B. Carter and A. I. Sanda, Phys. Rev. D 23, 1567 (1981). I. I. Y. Bigi and A. I. Sanda, Nucl. Phys. B 193, 85 (1981).

153. A. Ali, E. Lunghi and A. Y. Parkhomenko, Eur. Phys. J. C 36, 183 (2004). [hep-ph/0403275].

154. M. Ciuchini, E. Franco, G. Martinelli, M. Pierini and L. Silvestrini, Prepared for 32nd International Conference on High-Energy Physics (ICHEP 04), Beijing, China, 16-22 Aug 2004.

155. C. W. Bauer, D. Pirjol, I. Z. Rothstein and I. W. Stewart, Phys. Rev. D 70, 054015 (2004) [hep-ph/0401188].

156. C. W. Bauer, I. Z. Rothstein and I. W. Stewart, Phys. Rev. D 74, 034010 (2006) [arXiv:hep-ph/0510241].

157. M. Gronau and D. Wyler, Phys. Lett. B 265, 172 (1991).

158. M. Gronau and D. London., Phys. Lett. B 253, 483 (1991).

159. D. Atwood, I. Dunietz and A. Soni, Phys. Rev. Lett. 78, 3257 (1997) [hep$\mathrm{ph} / 9612433]$.

160. D. Atwood, I. Dunietz and A. Soni, Phys. Rev. D 63, 036005 (2001) [hep$\mathrm{ph} / 0008090]$.

161. M. Gronau, Phys. Lett. B 557, 198 (2003) [hep-ph/0211282].

162. D. Atwood and A. Soni, Phys. Rev. D 71, 013007 (2005) [arXiv:hep$\mathrm{ph} / 0312100]$.

163. A. Giri, Y. Grossman, A. Soffer and J. Zupan, Phys. Rev. D 68, 054018 (2003) [hep-ph/0303187].

164. A. J. Buras, R. Fleischer, S. Recksiegel and F. Schwab, Acta Phys. Polon. B 36, 2015 (2005) [arXiv:hep-ph/0410407]; Nucl. Phys. B 697, 133 (2004) [hep-ph/0402112]; Phys. Rev. Lett. 92, 101804 (2004) [hep-ph/0312259].

165. R. Fleischer and T. Mannel, Phys. Rev. D 57, 2752 (1998) [hep-ph/9704423]. 166. M. Neubert and J. L. Rosner, Phys. Rev. Lett. 81, 5076 (1998) [hep- 
ph/9809311]; Phys. Lett. B 441, 403 (1998) [hep-ph/9808493].

167. H. J. Lipkin, Phys. Lett. B 445, 403 (1999) [hep-ph/9810351].

168. A. J. Buras and R. Fleischer, Eur. Phys. J. C 16, 97 (2000) [hepph/0003323]; Eur. Phys. J. C 11, 93 (1999) [hep-ph/9810260].

169. T. Yoshikawa, Phys. Rev. D 68, 054023 (2003) [arXiv:hep-ph/0306147]; J. Korean Phys. Soc. 45, S479 (2004) [arXiv:hep-ph/0312097].

170. M. Beneke and M. Neubert, Nucl. Phys. B 675, 333 (2003). [hep$\mathrm{ph} / 0308039]$.

171. R. Fleischer and S. Recksiegel, Eur. Phys. J. C 38, 251 (2004) [arXiv:hep$\mathrm{ph} / 0408016]$.

172. M. Beneke, G. Buchalla, C. Greub, A. Lenz and U. Nierste, Phys. Lett. B 459, 631 (1999) [arXiv:hep-ph/9808385].

173. V. M. Abazov et al. [D0 Collaboration], Phys. Rev. Lett. 98, 121801 (2007) [arXiv:hep-ex/0701012].

174. T. Kuhr [CDF Collaboration], arXiv:0710.1789 [hep-ex].

175. For a recent review of $\mathrm{CP}$ violation at the LHC, see R. Fleischer, In the Proceedings of International Conference on Heavy Quarks and Leptons (HQL 06), Munich, Germany, 16-20 Oct 2006, pp 020 [arXiv:hep-ph/0703112].

176. O. Schneider, LHCb Upgrade Workshop, Edinburgh, January 11 -12, 2007.

177. P. Mack [CDF Collaboration], arXiv:0710.2502 [hep-ex].

178. D0 Public Note 5344-CONF based on $2 \mathrm{fb}^{-1}$ data, submitted to the EPS Conference, 2007.

179. G. Buchalla and A. J. Buras, Nucl. Phys. B 400, 225 (1993); ibid. 548, 309 (1999) [hep-ph/9901288]; M. Misiak and J. Urban, Phys. Lett. B 451, 161 (1999) [hep-ph/9901278]; A. J. Buras, Phys. Lett. B 566, 115 (2003) [hep$\mathrm{ph} / 0303060]$.

180. R. Dermisek, S. Raby, L. Roszkowski and R. Ruiz De Austri, JHEP 0304, 037 (2003) [hep-ph/0304101].

181. J. R. Ellis, S. Heinemeyer, K. A. Olive and G. Weiglein, JHEP 0502, 013 (2005) [arXiv:hep-ph/0411216].

182. H. J. Lipkin, Phys. Lett. B 621, 126 (2005) [arXiv:hep-ph/0503022].

183. M. Beneke, J. Rohrer and D. Yang, Nucl. Phys. B774, 64 (2007) [hepph/0612290]; M. Beneke and M. Neubert, Nucl. Phys. B675, 333(2003) [hep$\mathrm{ph} / 0308039]$.

184. A. Williamson and J. Zupan, Phys. Rev. D74, 014003(2006); Erratum-ibid. D74, 039901 (2006) [hep-ph/0601214].

185. A. Ali, G. Kramer, Y. Li, C. D. Lu, Y. L. Shen, W. Wang and Y. M. Wang, Phys. Rev. D 76, 074018 (2007) [arXiv:hep-ph/0703162]. 